\title{
Circumstellar masers in the Magellanic Clouds
}

\author{
J. Th. van Loon ${ }^{1}$, A. A. Zijlstra ${ }^{2}$, V. Bujarrabal ${ }^{3}$, and L.-A. Nyman ${ }^{4,5}$ \\ 1 Institute of Astronomy, Madingley Road, Cambridge CB3 0HA, UK \\ 2 UMIST, PO Box 88, Manchester M60 1QD, UK \\ 3 Observatorio Astronómico Nacional, Campus Universitario, Apartado 1143, 28800 Alcalá de Henares, Spain \\ 4 European Southern Observatory, Casilla 19001, Santiago 19, Chile \\ 5 Onsala Space Observatory, 43992 Onsala, Sweden
}

Received 9 October 2000 / Accepted 3 January 2001

\begin{abstract}
Results are presented of a search for $22 \mathrm{GHz} \mathrm{H}_{2} \mathrm{O} 6_{16} \rightarrow 5_{23}, 43 \mathrm{GHz} \mathrm{SiO}_{v=1}(J=1 \rightarrow 0), 86 \mathrm{GHz}$ $\mathrm{SiO}_{v=1}(J=2 \rightarrow 1)$ and $129 \mathrm{GHz} \mathrm{SiO}_{v=1}(J=3 \rightarrow 2)$ maser emission from bright IRAS point sources in the Small and Large Magellanic Clouds - mostly circumstellar envelopes around obscured red supergiants and Asymptotic Giant Branch stars (OH/IR stars). The aim of this effort was to test whether the kinematics of the mass loss from these stars depends on metallicity. $\mathrm{H}_{2} \mathrm{O}$ maser emission was detected in the red supergiants IRAS 04553-6825 and IRAS 05280-6910, and tentatively in the luminous IR object IRAS 05216-6753 and the AGB star IRAS 05329-6708, all in the $\mathrm{LMC} \mathrm{SiO}_{v=1}(J=2 \rightarrow 1)$ maser emission was detected in IRAS 04553-6825. The double-peaked $\mathrm{H}_{2} \mathrm{O}$ maser line profiles of IRAS 04553-6825 and IRAS 05280-6910, in combination with the $\mathrm{OH}$ (and $\mathrm{SiO}$ ) maser line profiles, yield the acceleration of the outflows from these stars. The outflow velocity increases between the $\mathrm{H}_{2} \mathrm{O}$ masing zone near the dust-formation region and the more distant $\mathrm{OH}$ masing zone from $v \sim 18$ to $26 \mathrm{~km} \mathrm{~s}^{-1}$ for IRAS 04553-6825 and from $v \sim 6$ to $17 \mathrm{~km} \mathrm{~s}^{-1}$ for IRAS 05280-6910. The total sample of LMC targets is analysed in comparison with circumstellar masers in the Galactic Centre. The photon fluxes of circumstellar masers in the LMC are found to be very similar to those in the Galactic Centre. The expansion velocities in the LMC appear to be $\sim 20 \%$ lower than for similarly bright OH masers in the Galactic Centre, but the data are still consistent with no difference in expansion velocity. OH/IR stars in the LMC appear to have slower accelerating envelopes than OH/IR stars in the Galactic Centre. The masers in the LMC have blue-asymmetric emission profiles. This may be due to the amplification of stellar and/or free-free radiation, rather than the amplification of dust emission, and may be more pronounced in low metallicity envelopes. The $\mathrm{SiO}$ maser strength increases with the photometric amplitude at $2.2 \mu \mathrm{m}$ but is independent of the photometric amplitude at $10 \mu \mathrm{m}$. This suggests a strong connection between shocks in the dust-free $\mathrm{SiO}$ masing zone and the dust formation process. The LMC masers obey the same trend as the Galactic Centre masers. Appendices describe $\mathrm{H}_{2} \mathrm{O}$ maser emission from the moderately mass-losing AGB star R Dor in the Milky Way, optical echelle spectroscopy of IRAS $04553-6825$, and the properties of circumstellar masers in the Galactic Centre.
\end{abstract}

Key words. masers - circumstellar matter - stars: mass loss - stars: AGB and post-AGB - supergiants Magellanic Clouds

\section{Introduction}

In the latest stages of their evolution, both massive and intermediate-mass stars pass through a phase of intense mass loss at rates of $10^{-6}$ to $10^{-3} M_{\odot} \mathrm{yr}^{-1}$ (van Loon et al. 1999b), returning a major fraction of their initial mass to the interstellar medium (ISM). For stars of $M_{\text {initial }} \gtrsim 8 M_{\odot}$ this occurs when they are red supergiants (RSGs), and for stars of $1 \lesssim M_{\text {initial }} \lesssim 8 M_{\odot}$ when they are Asymptotic Giant Branch (AGB) stars before becoming a Planetary Nebula (PN). They become enshrouded

Send offprint requests to: Jacco van Loon, e-mail: jacco@ast.cam.ac.uk by their dusty circumstellar envelope (CSE), rendering them invisible at optical wavelengths. The absorbed radiation is re-emitted by the dust at longer wavelengths, making them very bright IR objects. This is also when, in oxygen-rich CSEs, maser emission from $\mathrm{OH}, \mathrm{H}_{2} \mathrm{O}$ and $\mathrm{SiO}$ molecules may be observed. Hence these objects are known as $\mathrm{OH} / \mathrm{IR}$ stars.

The locations and intensities of masers are determined by molecular abundance, dust temperature, gas density, and velocity (Goldreich \& Scoville 1976; Lewis 1989). Where $\mathrm{H}_{2} \mathrm{O}$ is not shielded from interstellar UV radiation it is dissociated into $\mathrm{OH}$. $\mathrm{SiO}$ is depleted into dust grains except close to the star. Maser strengths scale with the 
local kinetic energy density or the local IR radiation field, which both depend on the CSE temperature. Masers are quenched above a critical density. Finally, the radiation field in the maser transition is amplified only by molecules that have small projected velocity differences (within the thermal width of the maser line, i.e. $\lesssim 1 \mathrm{~km} \mathrm{~s}^{-1}$ ).

As a result the CSE has a layered maser structure, corresponding to subsequent stages in the mass-loss process from the underlying star. Strong pulsations of the stellar photosphere of these Long Period Variables (LPVs) eject matter in which dust forms at typically 1 to 10 stellar radii $\left(R_{\star}\right)$. Radiation pressure accelerates the matter to velocities exceeding the escape velocity. Matter that does not reach the dust formation radius falls back to the star. At distances of $\gtrsim 100 R_{\star}$, the stellar wind flows with constant velocity $v_{\infty}$ into interstellar space. $\mathrm{SiO}$ masers probe the inner dust-free zone, $\mathrm{H}_{2} \mathrm{O}$ masers probe the acceleration zone, and $\mathrm{OH}$ masers probe the final stellar wind. This has been beautifully confirmed by interferometric observations of Galactic OH/IR stars (Diamond et al. 1984; Richards et al. 1996; Colomer et al. 2000).

The physical conditions in the CSE, and the evolutionary stage of the star can be determined from the presence or absence of different species of masers, and from their photon fluxes. The kinematic structure of the CSE can be determined from the maser line profiles. $\mathrm{SiO}$ maser radiation is predominantly amplified tangentially, resulting in a single maser peak within a few $\mathrm{km} \mathrm{s}^{-1}$ of the stellar velocity $v_{\star}$. OH maser radiation is beamed radially, resulting in a double-peaked line profile, spanning $2 \times v_{\infty} . \mathrm{H}_{2} \mathrm{O}$ masers are single-peaked in Mira variables but double-peaked in OH/IR stars (Takaba et al. 1994). Hence, in OH/IR stars $\mathrm{H}_{2} \mathrm{O}$ masers yield the expansion velocity at the base of the acceleration zone in the CSE.

A significant population of confirmed and suspected $\mathrm{OH} / \mathrm{IR}$ stars in the Small and Large Magellanic Clouds (SMC \& LMC) has now been identified and studied (Wood et al. 1992; Zijlstra et al. 1996; Loup et al. 1997; van Loon et al. 1997, 1998a, 1999a,b). The metallicities of the intermediate-age populations in the SMC and LMC are $\sim 7$ and $\sim 3 \times$ lower than solar, and hence the dependence of the mass loss on metallicity may be investigated (van Loon 2000). A study of Magellanic circumstellar masers may shed light on the metallicity dependence of the envelope kinematics. Here the final results are presented of surveys for $\mathrm{H}_{2} \mathrm{O}$ and $\mathrm{SiO}$ masers in the Magellanic Clouds (van Loon et al. 1996, 1998b).

\section{Radio observations}

A summary of the observations can be found in Table 1 . No attempts were made to better determine the positions of (tentatively) detected maser sources.

\section{1. $\mathrm{H}_{2} \mathrm{O}$ maser emission at $22 \mathrm{GHz}$ with Parkes}

The $64 \mathrm{~m}$ radio telescope at Parkes, Australia, was used with the $1.3 \mathrm{~cm}$ receiver plus autocorrelator to observe
$22 \mathrm{GHz} \mathrm{H}_{2} \mathrm{O}$ masers. Using the Dual Circular feed spectra were obtained simultaneously in left and right circular polarization. No difference was found between them, and they were averaged. The current $K$-band facility at Parkes is not as powerful as the beam-switching set-up used in the early 1980's that yielded $T_{\mathrm{sys}} \sim 90 \mathrm{~K}$ (Whiteoak \& Gardner 1986). Weather conditions were generally fair.

In the 1997 run only the LMC targets IRAS 04553-6825 and IRAS 05329-6708 were observed, for six and three hours on-source integration, respectively. The nearby sky was measured every two minutes, resulting in very flat baselines that required only a shallow second-order polynomial to be subtracted. The $22 \mathrm{GHz}$ discovery spectrum of IRAS 04553-6825 was already presented in van Loon et al. (1998b). In the 2000 run, on-source integration times were a few hours per target. The sky was measured every two minutes most of the time at $5^{\prime} \mathrm{N}$ of the source. Pointing, focus and calibration were checked regularly by observing the nearby bright maser source R Dor (see Appendix A) at different zenith distances. The pointing accuracy was found to be $\sim 10^{\prime \prime}$ and flux losses due to pointing errors are less than $\sim 10 \%$.

\section{2. $\mathrm{H}_{2} \mathrm{O}$ maser emission at $22 \mathrm{GHz}$ with Mopra}

The $22 \mathrm{~m}$ radio telescope at Mopra, Australia, was used with the $1.3 \mathrm{~cm}$ receiver plus autocorrelator to observe $22 \mathrm{GHz} \mathrm{H}_{2} \mathrm{O}$ masers. The opacity correction was usually between 1.3 and 1.6. The conversion factor agrees with the observed noise and flux density for R Dor (48 Jy).

The background was measured every four minutes at both sides of the target. The baseline was estimated for each individual spectrum by a running average (within 1- $\sigma$ around the median) over 55 channels. After subtracting the baselines, the flux density was obtained by averaging (within 3- $\sigma$ around the median) over the time series of spectra. This procedure yielded flat baselines and effectively rejected bad spectra. Flux conservation was tested on the well-detected $\mathrm{H}_{2} \mathrm{O}$ maser spectra of IRAS 04553-6825.

\subsection{SiO maser emission at $43 \mathrm{GHz}$ with Parkes}

The $64 \mathrm{~m}$ radio telescope at Parkes, Australia, was used with the $0.7 \mathrm{~cm}$ single polarization receiver plus correlator to observe $43 \mathrm{GHz} \mathrm{SiO}$ masers. The background was measured by alternation between two targets every 150 seconds. Reasonably flat baselines were obtained by subtracting a fifth order polynomial plus a sine with a period of 200 channels. The useful heliocentric velocity range runs from 167 to $322 \mathrm{~km} \mathrm{~s}^{-1}$.

\subsection{SiO maser emission at $86 \mathrm{GHz}$ with Mopra}

The $22 \mathrm{~m}$ radio telescope at Mopra, Australia, was used with the $3 \mathrm{~mm}$ SIS receiver plus correlator to observe 
Table 1. Summary of observations: observatory, dates, transition, rest frequency, centre velocity, channel velocity width, number of channels, bandwidth, effective telescope diameter, beam Full-Width-Half-Maximum, typical system temperature and conversion factor. See text for more SEST observations

\begin{tabular}{|c|c|c|c|c|c|c|c|c|c|c|c|}
\hline site & $\begin{array}{r}\text { dates } \\
\mathrm{d} / \mathrm{m} / \mathrm{y}\end{array}$ & transition & $\begin{array}{l}\nu_{0} \\
\mathrm{GHz}\end{array}$ & $\begin{array}{l}v_{\text {hel,c }} \\
\mathrm{km} \mathrm{s}^{-1}\end{array}$ & $\begin{array}{l}\Delta v \\
\mathrm{~km} \mathrm{~s}^{-1}\end{array}$ & $\bar{N}$ & $\begin{array}{l}\text { band } \\
\mathrm{MHz}\end{array}$ & $\begin{array}{l}D_{\text {eff }} \\
\mathrm{m}\end{array}$ & beam & $\begin{array}{c}T_{\text {sys }} \\
\mathrm{K}\end{array}$ & $\begin{array}{r}\mathrm{cal} . \\
\mathrm{Jy} / \mathrm{K}\end{array}$ \\
\hline Parkes & $19-20 / 8 / 97$ & $\mathrm{H}_{2} \mathrm{O} 6_{16} \rightarrow 5_{23}$ & 22.23507985 & 340 & 0.84 & 1024 & 64 & 45 & 1.3 & 110 & 6 \\
\hline Parkes & $5-13 / 4 / 00$ & $\mathrm{H}_{2} \mathrm{O} 6_{16} \rightarrow 5_{23}$ & 22.23507985 & 200 & 0.42 & 2049 & 64 & 45 & 1.3 & $140-180$ & 6 \\
\hline Mopra & $18-29 / 1 / 99$ & $\mathrm{H}_{2} \mathrm{O} 6_{16} \rightarrow 5_{23}$ & 22.23507985 & 270 & 0.84 & 1024 & 64 & 22 & 2.7 & 115 & 20 \\
\hline Parkes & $16-25 / 8 / 95$ & $\mathrm{SiO} 1 \rightarrow 0(v=1)$ & 43.122080 & 249.8 & 0.2173 & 1024 & 32 & 17 & 1.6 & 80 & 39 \\
\hline Mopra & $29 / 8-6 / 9 / 95$ & $\mathrm{SiO} 2 \rightarrow 1(v=1)$ & 86.243442 & 250 & 0.2173 & 1024 & 64 & 16 & 1.0 & 130 & 45 \\
\hline SEST & $25-31 / 5 / 95$ & $\mathrm{SiO} 2 \rightarrow 1(v=1)$ & 86.243442 & 290 & 0.15 & 2000 & 86 & 15 & 0.95 & $110-150$ & 25 \\
\hline SEST & $29-31 / 5 / 95$ & $\mathrm{SiO} 2 \rightarrow 1(v=1)$ & 86.243442 & 290 & 2.4 & 1600 & 1086 & 15 & 0.95 & $110-150$ & 25 \\
\hline SEST & $25-28 / 5 / 95$ & $\mathrm{SiO} 3 \rightarrow 2(v=1)$ & 129.363368 & 290 & 1.6 & 1440 & 995 & 15 & 0.67 & $190-250$ & 29 \\
\hline
\end{tabular}

$86 \mathrm{GHz} \mathrm{SiO}$ masers. The spectra were obtained simultaneously in two orthogonal polarizations that were then averaged. The pointing was checked every few hours on $\mathrm{R}$ Dor, and was accurate to $<15^{\prime \prime} \mathrm{rms}$. The background was measured by alternation between two of these targets every few minutes. The baselines were very flat.

\subsection{SiO maser emission at 86 \& $129 \mathrm{GHz}$ with SEST}

The $15 \mathrm{~m}$ Swedish-ESO Sub-mm Telescope (SEST) at the European Southern Observatory (ESO) at La Silla, Chile, was used with the $3 \mathrm{~mm}$ receiver plus the acousto-optical High Resolution Spectrograph (HRS) to observe $86 \mathrm{GHz}$ $\mathrm{SiO}$ masers. This configuration was used simultaneously with the Low Resolution Spectrograph (LRS) either tuned at the same frequency or coupled to the $2 \mathrm{~mm}$ receiver to observe $129 \mathrm{GHz} \mathrm{SiO}$ masers.

The internal absolute flux calibration is accurate to $\sim 20 \%$. The observations were done in Double Beam Switch Mode, with a beam throw of $\sim 11.5^{\prime}$ in azimuth. The pointing was checked every few hours on $\mathrm{R}$ Dor, and was accurate to $\sim 3^{\prime \prime} \mathrm{rms}$. The atmospheric conditions were very good: a relative humidity of typically 15 to $30 \%$, an outside air temperature of $\sim 15^{\circ} \mathrm{C}$, and little or no cirrus. The baselines were very flat, requiring only a zeroth order polynomial to be subtracted from each spectrum.

IRAS 04553-6825, after its $86 \mathrm{GHz} \mathrm{SiO}$ maser emission had been detected (van Loon et al. 1996), was reobserved on 7 occasions during the period from July 1996 to January 1998, with the SEST and the HRS and LRS at $86 \mathrm{GHz}$ using the same observing strategy as described above. The total on-source integration time at $86 \mathrm{GHz}$ was 65 and $51 \mathrm{hr}$ with the HRS and LRS, respectively. During this campaign IRAS 05329-6708 and IRAS 05280-6910 were re-observed in January 1998.

\section{Results from the maser searches}

The targets comprise the known Magellanic OH/IR stars, plus other IR objects in the SMC and LMC: mainly dustenshrouded AGB stars, RSGs or H II emission objects (LHA numbers from Henize 1956) often related to sites of recent/on-going star formation. Additional $22 \mathrm{GHz}$ ob-
Table 2. The SMC \& LMC targets, together with their IRAS flux densities (in Jy), classifications and whether $\mathrm{OH} 1612 \mathrm{MHz}$ masers are detected (yes), not detected (no), or not tried ( )

\begin{tabular}{lcrlc}
\hline \hline IRAS-PSC & $S_{12}$ & \multicolumn{1}{c}{$S_{25}$} & type & OH \\
\hline SMC sources & & & & \\
$00483-7347$ & 0.64 & 0.49 & AGB & \\
$00486-7308^{\dagger}$ & 0.41 & $<0.60$ & AGB & \\
$01074-7140$ & 0.36 & 0.47 & AGB & \\
\hline LMC sources & & & & \\
$04407-7000$ & 0.81 & 0.70 & AGB & yes \\
$04491-6915$ & 0.53 & 2.17 & H II & \\
$04498-6842$ & 1.31 & 1.05 & AGB & \\
$04514-6931$ & 0.36 & 3.52 & H II $?$ & \\
$04530-6916$ & 2.12 & 5.10 & RSG ? & no \\
$04545-7000$ & 0.52 & 0.84 & AGB & yes \\
$04546-6915$ & 1.17 & 9.67 & H II & \\
$04553-6933$ & 0.53 & 0.48 & RSG & \\
$04553-6825$ & 9.15 & 14.36 & RSG & yes \\
$04571-6627$ & 0.42 & 3.03 & H II & \\
$04581-7013$ & 0.41 & 0.39 & RSG & \\
$05198-6941$ & 2.63 & 7.15 & H II & \\
$05216-6753$ & 4.10 & 14.56 & RSG ? & no \\
$05280-6910$ & 4.16 & 24.18 & RSG ? & yes \\
$05298-6957$ & 0.85 & 1.38 & AGB & yes \\
$05325-6743$ & 1.20 & 6.47 & H II & \\
$05329-6708$ & 0.98 & 1.48 & AGB & yes \\
$05346-6949$ & 7.78 & 20.75 & RSG & no \\
$05402-6956$ & 0.82 & 1.11 & AGB & yes \\
\hline
\end{tabular}

${ }^{\dagger}$ IRAS 00486-7308 is from the IRAS-FSC with flux densities from Groenewegen \& Blommaert (1998).

servations of the Galactic AGB star R Dor are described in Appendix A. The IRAS names of the targets are listed in Table 2, together with the IRAS flux densities at 12 and $25 \mu \mathrm{m}$, object classification, and whether $\mathrm{OH}$ maser emission has been detected (yes), not detected (no), or not tried ( ) by Wood et al. $(1986,1992)$ or van Loon et al. (1998a). The results for the targets (tentatively) detected in at least one of the $\mathrm{H}_{2} \mathrm{O}$ or $\mathrm{SiO}$ maser transitions are summarised in Table 3, whilst upper limits (3- $\sigma$ noise levels) for the non detections are summarised in Table 4. 
Table 3. Peak flux densities ( $\pm 1-\sigma$, in Jy) for objects detected (boldface) in a $\mathrm{H}_{2} \mathrm{O}$ and/or $\mathrm{SiO}$ maser transition: $\mathrm{H}_{2} \mathrm{O} 22 \mathrm{GHz}$ with Parkes (P; 1997 \& 2000) and Mopra (M), SiO 43 GHz with Parkes, SiO 86 GHz with Mopra and SEST (S; HRS \& LRS), and $\mathrm{SiO} 129 \mathrm{GHz}$ with SEST. Tentative detections are between parentheses, 3- $\sigma$ upper limits are given for non detections

\begin{tabular}{|c|c|c|c|c|c|c|c|c|}
\hline \multirow[b]{2}{*}{ IRAS-PSC } & \multicolumn{3}{|c|}{$\overline{\mathrm{H}} \mathrm{H}_{2} \mathrm{O}$} & \multicolumn{5}{|c|}{$\mathrm{SiO}$} \\
\hline & $P_{22}(1997)$ & $P_{22}(2000)$ & $M_{22}$ & $P_{43}$ & $M_{86}$ & $S_{86}(\mathrm{HRS})$ & $S_{86}(\mathrm{LRS})$ & $S_{129}$ \\
\hline LMC sources & & & & & & & & \\
\hline $04553-6825$ & $0.068 \pm 0.006$ & $0.195 \pm 0.010$ & $0.20 \pm 0.04$ & $<0.67$ & $<0.22$ & $0.150 \pm 0.025$ & $0.072 \pm 0.011$ & $<0.15$ \\
\hline $05216-6753$ & & $(0.046 \pm 0.020)$ & & $<0.90$ & & $<0.28$ & $<0.16$ & \\
\hline $05280-6910$ & & $0.137 \pm 0.010$ & $(0.30 \pm 0.06)$ & $<1.5$ & $<0.34$ & $(0.30 \pm 0.08)$ & $<0.22$ & $<0.18$ \\
\hline $05329-6708$ & $(0.026 \pm 0.007)$ & $<0.027$ & $<0.09$ & $<1.6$ & & $(0.12 \pm 0.07)$ & $<0.14$ & $<0.17$ \\
\hline
\end{tabular}

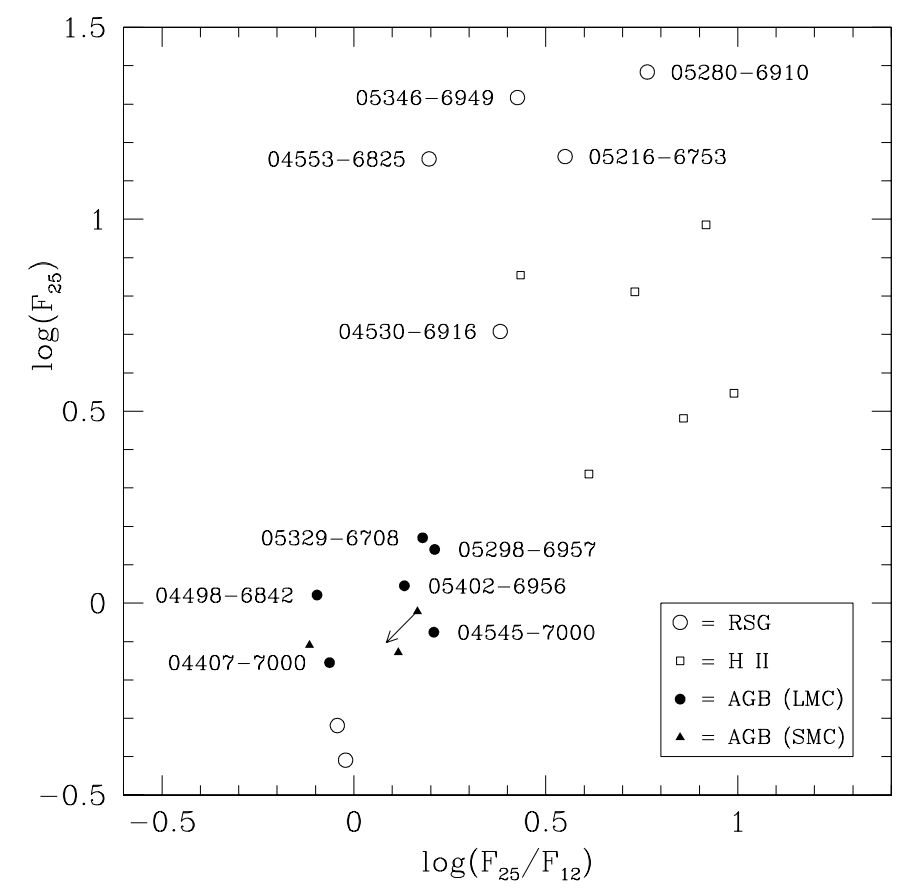

Fig. 1. Diagram of $25 \mu \mathrm{m}$ flux density versus 25 over $12 \mu \mathrm{m}$ flux density ratio for the targets of the maser search, distinguished according to their object classes (RSGs, H II regions, AGB stars). The flux densities of the SMC objects have been scaled by +0.2 dex to the distance of the LMC

The IRAS mid-IR flux densities and flux density ratios often carry information about the nature of the IR source (Fig. 1). The AGB stars amongst the targets represent the most luminous AGB stars with the highest mass-loss rates encountered in the MCs, with typical mid-IR flux densities of $\sim 1$ Jy. Because of their higher luminosity and sometimes also higher mass-loss rate, RSGs may become somewhat redder and more than an order of magnitude brighter at $25 \mu \mathrm{m}$ than these extreme AGB stars. RSGs, due to their short lifetimes, may still be associated with $\mathrm{H}$ II regions that usually have very red mid-IR colours. Very young supergiants may be dust-enshrouded inside of an ultracompact H II region with similarly red colours (see, for instance, Persi et al. 1994). The individual targets are described below - with a general reference to Loup et al. (1997) - together with the results from our maser search.
Table 4. Upper limits (3- $\sigma$ noise levels, in Jy) for the non detections: $\mathrm{H}_{2} \mathrm{O} 22 \mathrm{GHz}$ with Parkes (P, 2000) and Mopra (M), $\mathrm{SiO} 43 \mathrm{GHz}$ with Parkes, and $\mathrm{SiO} 86 \mathrm{GHz}$ with Mopra

\begin{tabular}{l|ll|ll}
\hline \hline & \multicolumn{2}{|c|}{$\mathrm{H}_{2} \mathrm{O}$} & \multicolumn{2}{c}{$\mathrm{SiO}$} \\
IRAS-PSC & $P_{22}(2000)$ & $M_{22}$ & $P_{43}$ & $M_{86}$ \\
\hline SMC sources & & & & \\
$00483-7347$ & 0.030 & 0.15 & & \\
$00486-7308$ & 0.033 & & & \\
$01074-7140$ & 0.039 & & & \\
\hline LMC sources & & & & \\
$04407-7000$ & & 0.09 & 1.5 & 0.35 \\
$04491-6915$ & & & 1.5 & \\
$04498-6842$ & & & 2.0 & \\
$04514-6931$ & & & 2.0 & \\
$04530-6916$ & 0.039 & 0.12 & & \\
$04545-7000$ & 0.027 & & 2.2 & \\
$04546-6915$ & & & 2.2 & \\
$04553-6933$ & & & 2.0 & \\
$04571-6627$ & & & 2.0 & \\
$04581-7013$ & & & 2.0 & \\
$05198-6941$ & & & 2.0 & \\
$05298-6957^{\dagger}$ & 0.024 & 0.17 & 1.2 & 0.46 \\
$05325-6743$ & & & 1.6 & \\
$05346-6949$ & 0.033 & 0.11 & 1.5 & \\
$05402-6956$ & 0.036 & 0.15 & 1.2 & \\
\hline
\end{tabular}

${ }^{\dagger}$ Observed with SEST at $86 \mathrm{GHz}$ (HRS \& LRS) and $129 \mathrm{GHz}$, with upper limits of $0.29,0.09$ and $0.17 \mathrm{Jy}$, respectively.

\subsection{RSGs in the $L M C$}

\subsubsection{IRAS 04530-6916}

This source may be interpreted as a luminous RSG or AGB star, with a high mass-loss rate of $\dot{M} \sim$ $810^{-4} M_{\odot} \mathrm{yr}^{-1}$ (van Loon et al. 1999b) and detected by IRAS even at $100 \mu \mathrm{m}$ (Trams et al. 1999). It is located in the $\mathrm{H}$ II region DEM L15. Variability in the near-IR is reported by Wood et al. (1992), with a period of $\sim 1260$ days, but the amplitudes are only $\lesssim 0.4$ mag even in the $J$-band. An $I$-band spectrum of the near-IR object associated with the IRAS source reveals an early-type emission-line spectrum (Cioni et al., in preparation) adding some confusion with regard to the nature of this object.

A narrow $3-\sigma$ peak $(\sim 150 \mathrm{mJy})$ at $v_{\text {hel }} \sim 200 \mathrm{~km} \mathrm{~s}^{-1}$ in the Mopra $22 \mathrm{GHz}$ spectrum could not be confirmed at 


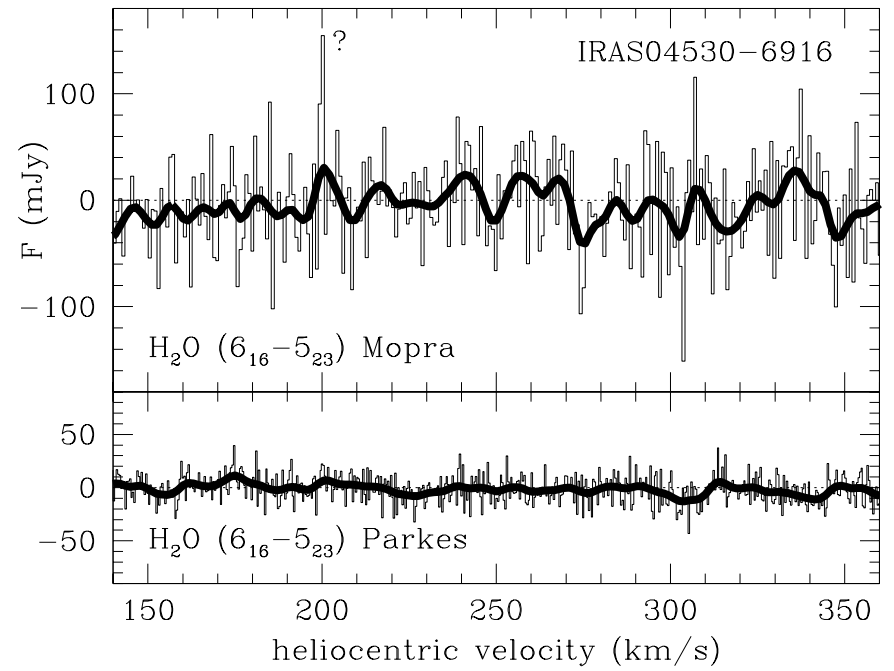

Fig. 2. IRAS 04530-6916: Mopra \& Parkes (2000) $22 \mathrm{GHz}$ spectra. The velocities are heliocentric. The boldfaced curves are the spectra smoothed by a Gaussian of $\sigma=2.0 \mathrm{~km} \mathrm{~s}^{-1}$. A possible $\mathrm{H}_{2} \mathrm{O}$ maser peak at $v_{\text {hel }} \sim 200 \mathrm{~km} \mathrm{~s}^{-1}$ in the Mopra spectrum could not be confirmed at Parkes despite the much lower noise levels

Parkes at much lower noise levels (Fig. 2), possibly due to temporal variability of the $\mathrm{H}_{2} \mathrm{O}$ maser emission (see, for instance, Persi et al. 1994) or to beamsize differences if the source is at $\sim 2^{\prime}$ from IRAS 04530-6916. The maser velocity would be rather different from the $21 \mathrm{~cm} \mathrm{H}$ I that peaks at $v_{\text {hel }} \sim 260 \mathrm{~km} \mathrm{~s}^{-1}$ (Kim et al. 1999), but this is also the case for some of the $\mathrm{H}_{2} \mathrm{O}$ masers in the giant star forming region 30 Doradus (van Loon \& Zijlstra 2000).

\subsubsection{IRAS 04553-6933}

This supergiant is of spectral type M2 (WOH S71: Westerlund et al. 1981).

\subsubsection{IRAS 04553-6825}

IRAS 04553-6825 is a very luminous obscured RSG that has been extensively discussed in the past (van Loon et al. 1999a,b and references therein). OH maser emission at 1612 and $1665 \mathrm{MHz}$ was discovered by Wood et al. (1986, 1992), $\mathrm{SiO}$ maser emission at $86 \mathrm{GHz}$ by van Loon et al. (1996), and $\mathrm{H}_{2} \mathrm{O}$ maser emission at $22 \mathrm{GHz}$ by van Loon et al. (1998b).

The $\mathrm{H}_{2} \mathrm{O}$ maser emission from IRAS 04553-6825 was confirmed with Mopra, and re-observed with Parkes on two occasions in the 2000 run. The (integration time \& system temperature weighted) average of the latter is presented in Fig. 3 (top panel). The integrated flux of the $\mathrm{H}_{2} \mathrm{O}$ maser emission between 255 and $307 \mathrm{~km} \mathrm{~s}^{-1}$ equals $0.65 \mathrm{Jy} \mathrm{kms}^{-1}$, corresponding to a photon flux of $1.010^{45} \mathrm{~s}^{-1}$. The very narrow main peak is brighter in the 2000 run at least partly due to the higher spectral resolution, and may, in fact, still be unresolved. The double peak at blue-shifted velocities is intriguing, as the

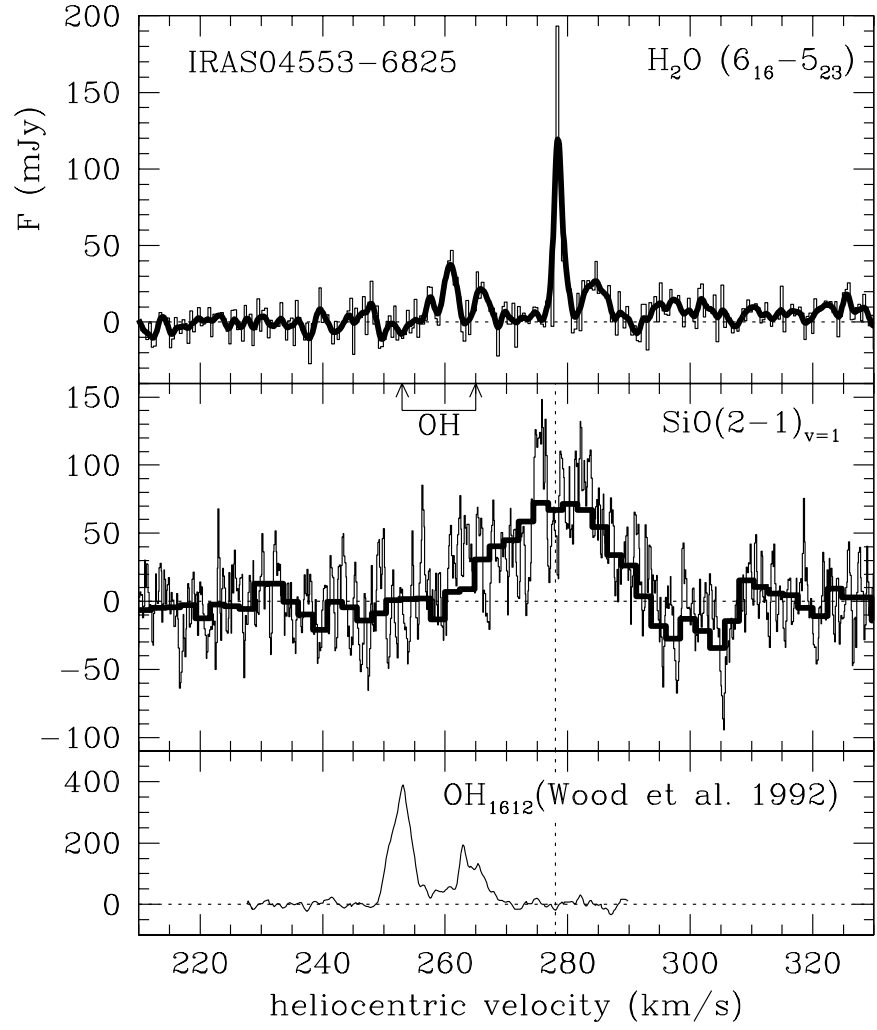

Fig. 3. IRAS 04553-6825: spectra of the $22 \mathrm{GHz} \mathrm{H}_{2} \mathrm{O}$ (top; Parkes 2000) and $86 \mathrm{GHz} \mathrm{SiO}$ (middle; SEST) maser emission. The boldfaced curve in the upper panel is the spectrum smoothed by a Gaussian of $\sigma=0.5 \mathrm{~km} \mathrm{~s}^{-1}$, whilst in the middle panel it is the LRS spectrum. The velocities are heliocentric. Also indicated are the velocities of the $1612 \mathrm{MHz} \mathrm{OH}$ maser peaks (arrows) and the velocity of the $\mathrm{H}_{2} \mathrm{O}$ maser peak (vertical dotted line). The $1612 \mathrm{MHz}$ spectrum of Wood et al. (1992) is plotted in the lower panel for comparison

shape closely resembles that of the $1612 \mathrm{MHz} \mathrm{OH}$ emission profile (Wood et al. 1992) but at slightly smaller velocity displacements of -13 and $-18 \mathrm{~km} \mathrm{~s}^{-1}$ with respect to the main $\mathrm{H}_{2} \mathrm{O}$ maser peak at $v_{\text {hel }}=278.5 \mathrm{~km} \mathrm{~s}^{-1}$, instead of -14 and $-26 \mathrm{~km} \mathrm{~s}^{-1}$. This strongly suggests that the blue-shifted $\mathrm{H}_{2} \mathrm{O}$ maser peaks and the $\mathrm{OH}$ maser peaks are formed in related but displaced regions in the CSE outflow. The blue-shifted $\mathrm{H}_{2} \mathrm{O}$ maser emission was not noticed in the discovery spectrum (van Loon et al. 1998b) but can be recovered in that data a posteriori. The additional redshifted $\mathrm{H}_{2} \mathrm{O}$ maser peak perhaps arises from some local density enhancement or increased coherent path length in part of the CSE at the far side of the star.

The composite spectrum of all SEST observations at $86 \mathrm{GHz}$ of IRAS $04553-6825$ is presented in Fig. 3 (middle panel). The HRS spectrum is overlaid by the LRS spectrum. The quality of the individual spectra is insufficient for a detailed study of spectral changes with pulsational phase: no variability more than a factor two is seen in the peak or integrated flux density. The $\mathrm{SiO}$ maser properties have been listed in van Loon et al. (1998b). SiO maser emission can be traced between velocities of $\sim 260$ and $290 \mathrm{~km} \mathrm{~s}^{-1}$, and there is evidence for the peak of the 
emission to be split into two peaks $\sim 8 \mathrm{kms}^{-1}$ apart. The shoulder of $\mathrm{SiO}$ maser emission between $\sim 260$ and $266 \mathrm{~km} \mathrm{~s}^{-1}$ may be related to the blue-shifted $\mathrm{H}_{2} \mathrm{O}$ maser peaks. The velocity of the main $\mathrm{H}_{2} \mathrm{O}$ maser peak falls right in the centre of the double-peaked $\mathrm{SiO}$ emission.

Two lines at 298 and $305 \mathrm{kms}^{-1}$ are detected apparently in absorption (Fig. 3, middle panel), and they are also visible at several individual epochs. $\mathrm{SiO}$ masing lines have never been seen in absorption in Galactic sources for good reasons: the excitation temperature is $\sim 1800 \mathrm{~K}$. The features may correspond to $\mathrm{SiO}$ emission in the off-source beam. It is hard to trace back this source as the beamswitching was done in azimuth and hence the off-source beam swept across the sky during each observing session.

\subsubsection{IRAS $04581-7013$}

This is a variable star (HV2255) of spectral type M4.

\subsubsection{IRAS 05216-6753}

This object is very bright in the IR, with possibly an earlytype supergiant underlying its CSE (Zijlstra et al. 1996). Marginal variability is detected by Wood et al. (1992), who suggest it may be a proto-PN or post-RSG object and who note the similarity with IRAS 04530-6916.

The $22 \mathrm{GHz}$ spectrum with Parkes shows a peak of $\sim 45 \mathrm{mJy}-$ a 3 to $4-\sigma$ level after some smoothing - at $v_{\text {hel }} \sim$ $284 \mathrm{~km} \mathrm{~s}^{-1}$ (Fig. 4). The peak has a FWHM of $\sim 3 \mathrm{~km} \mathrm{~s}^{-1}$. The integrated flux of the emission is $\sim 0.5 \mathrm{Jy} \mathrm{km} \mathrm{s}^{-1}$, corresponding to a photon flux of $\sim 810^{44} \mathrm{~s}^{-1}$. There may be a secondary peak at $289 \mathrm{kms}^{-1}$.

\subsection{IRAS 05280-6910}

The $1665 \mathrm{MHz}$ OH maser attributed to IRAS 05280-6910 is located at the centre of the cluster NGC 1984 (Wood et al. 1992). The IRAS 12, 25 and $60 \mu \mathrm{m}$ flux densities suggest a very cool and luminous CSE, possibly a postRSG object. The proposed near-IR counterpart is with $K=8.19 \mathrm{mag}$ very bright, but with $(J-K)=1.01$ not very red (Wood et al. 1992). Within the Parkes beam

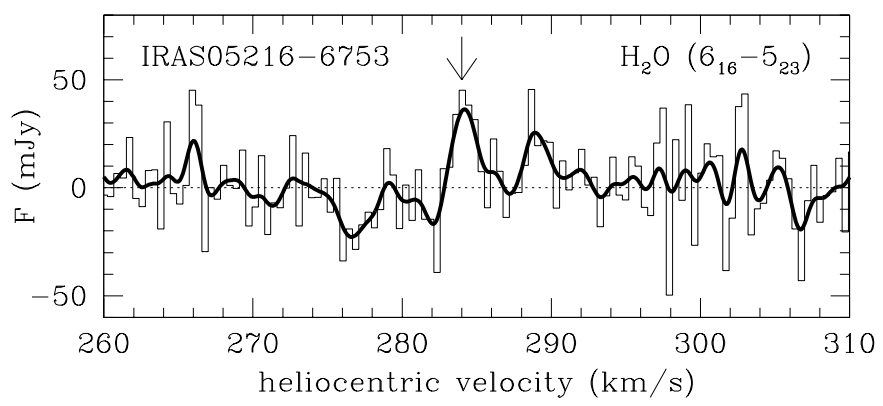

Fig. 4. IRAS 05216-6753: spectrum of the tentative detection of $22 \mathrm{GHz} \mathrm{H}_{2} \mathrm{O}$ maser emission (indicated by the arrow). The velocities are heliocentric. The boldfaced curve is the spectrum smoothed by a Gaussian of $\sigma=0.5 \mathrm{~km} \mathrm{~s}^{-1}$

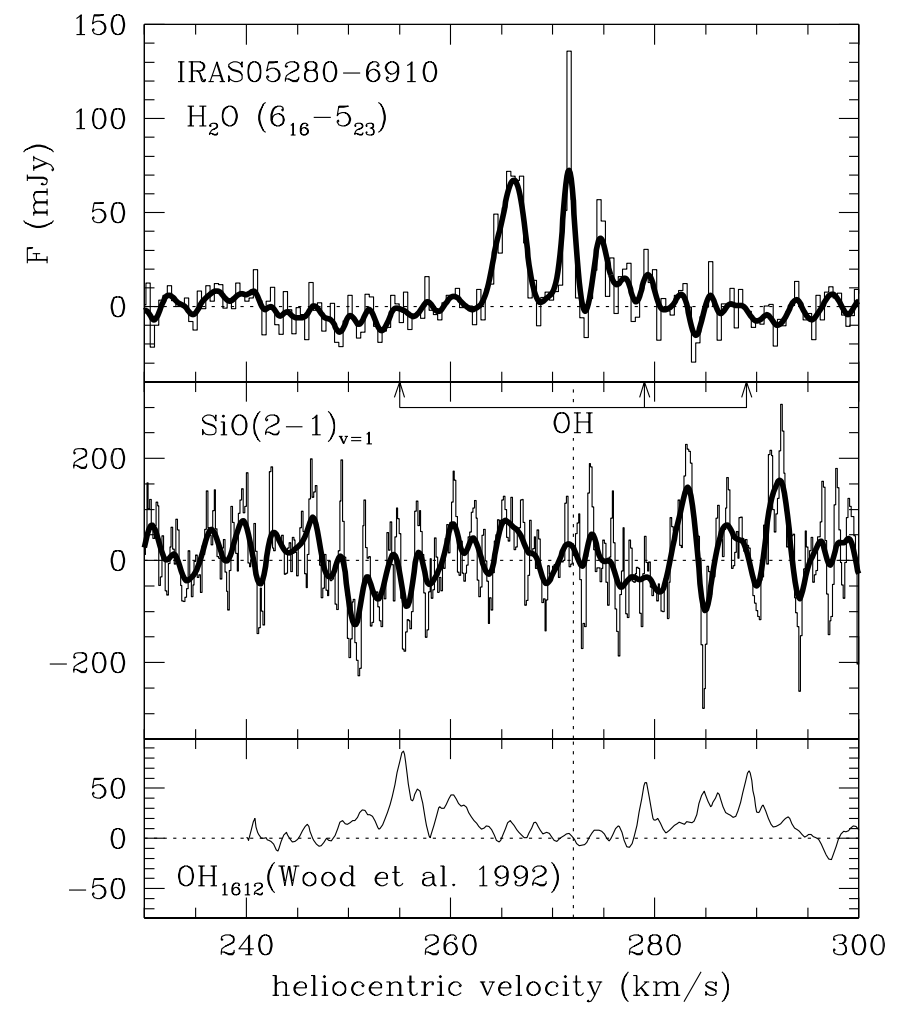

Fig. 5. IRAS 05280-6910: spectra of the $22 \mathrm{GHz} \mathrm{H}_{2} \mathrm{O}$ (top; Parkes 2000) and $86 \mathrm{GHz} \mathrm{SiO}$ (middle; SEST) maser emission. The boldfaced curves are the spectra smoothed by a Gaussian of $\sigma=0.5 \mathrm{~km} \mathrm{~s}^{-1}$. The velocities are heliocentric. Also indicated are the velocities of the $1612 \mathrm{MHz} \mathrm{OH}$ maser peaks (arrows) and the velocity of the $\mathrm{H}_{2} \mathrm{O}$ maser peak (vertical dotted line). The $1612 \mathrm{MHz}$ spectrum of Wood et al. (1992) is plotted in the lower panel for comparison

of $\sim 13^{\prime}$ at $\mathrm{OH}$ frequencies are three luminous late type variable stars of $V \sim 13$ and $I \sim 10 \mathrm{mag}$, a PN (SMP LMC 64), several IR objects, the H II region DEM L198 at $4.2^{\prime} \mathrm{W}$, and the cluster NGC 1994 (IRAS 05287-6910) at $\sim 4^{\prime}$ E. It may well be that only the $1612 \mathrm{MHz}$ emission arises from the CSE of an evolved star in the region of NGC 1984, whilst the $1665 \mathrm{MHz}$ emission arises from the ISM.

Bright and complex $\mathrm{H}_{2} \mathrm{O}$ maser emission was detected with Parkes (Fig. 5, top panel). The very narrow and probably unresolved main peak is at $v_{\text {hel }}=272 \mathrm{~km} \mathrm{~s}^{-1}$, which also defines the centroid velocity of the $\mathrm{OH} 1612$ $\mathrm{MHz}$ maser emission as observed by Wood et al. (1992). This confirms the cluster object IRAS 05280-6910 to be the source of the $1612 \mathrm{MHz} \mathrm{OH}$ maser emission. A rather broad and bright additional peak is blue-shifted by $\sim 5 \mathrm{~km} \mathrm{~s}^{-1}$, and fainter and possibly sub-structured emission is seen at red-shifted velocities of $\sim 3$ to $8 \mathrm{~km} \mathrm{~s}^{-1}$ with respect to the main peak. The integrated flux of the $\mathrm{H}_{2} \mathrm{O}$ maser emission between 260 and $284 \mathrm{~km} \mathrm{~s}^{-1}$ equals $0.42 \mathrm{Jy} \mathrm{kms} \mathrm{km}^{-1}$, corresponding to a photon flux of $6.510^{44} \mathrm{~s}^{-1}$, not much less than IRAS 04553-6825. Both the main and the blue-shifted additional peaks can 
be recovered a posteriori in the Mopra $22 \mathrm{GHz}$ data of IRAS 05280-6910.

The $86 \mathrm{GHz} \mathrm{SiO}$ maser spectrum of IRAS 05280-6910 is presented in Fig. 5 (middle panel). A constant baseline offset of $2 \mathrm{mJy}$ has been subtracted. The strongest peak, at a heliocentric velocity of $292 \mathrm{~km} \mathrm{~s}^{-1}$, has a peak flux density of $0.30 \mathrm{Jy}(=3.8 \sigma)$. There is another $(2.8 \sigma)$ peak at a velocity of $284 \mathrm{~km} \mathrm{~s}^{-1}$, and a hint of faint emission at $v_{\text {hel }} \sim 265 \mathrm{~km} \mathrm{~s}^{-1}$ (at which also $\mathrm{H}_{2} \mathrm{O}$ maser emission is seen). The integrated flux of the emission around 265, 284 and $292 \mathrm{~km} \mathrm{~s}^{-1}$ equals $\sim 0.8 \mathrm{Jy} \mathrm{km} \mathrm{s}^{-1}$, corresponding to a photon flux of $1.210^{45} \mathrm{~s}^{-1}$. The $\mathrm{SiO}$ detection is rather uncertain, though, and might still be (strong) noise.

\subsubsection{IRAS 05346-6949}

This is thought to be a very luminous and highly obscured RSG (Elias et al. 1986), but this is still uncertain. Its IRAS-LRS spectrum is odd in that it shows a flat continuum with peculiar features (Kwok et al. 1997).

\section{3. $A G B$ stars in the $L M C$}

The AGB stars amongst the LMC targets are oxygen rich, very luminous, variable on long timescales $\left(\sim 10^{3}\right.$ days) and severely dust-enshrouded (Wood et al. 1992; van Loon et al. 1998a, 1999a,b). For some, mid-IR spectra have been obtained by Trams et al. (1999), showing the $10 \mu \mathrm{m}$ silicate dust feature in absorption with some emission wings remaining (see also Groenewegen et al. 1995; Zijlstra et al. 1996 for IRAS 05329-6708).

These stars comprise all known AGB sources of $\mathrm{OH}$ maser emission in the LMC, except the bluest amongst them, IRAS 04498-6842, that has not (yet) been detected at $1612 \mathrm{MHz}$. The only typical saturated double-peaked OH maser emission profile is found in IRAS 05298-6957. IRAS $04407-7000$ is single peaked at $1612 \mathrm{MHz}$ (van Loon et al. 1998a), and consequently no expansion velocity of the CSE can be determined, whilst the other emission profiles are rather complex and/or faint.

\subsubsection{IRAS 04545-7000}

This object is located in a small double association of stars (\#200 \& 201 in Bica et al. 1999), with a cluster at $\sim 2^{\prime}$.

\subsubsection{IRAS 05298-6957}

This star is in a cluster containing a carbon star and has an initial mass of $M_{\text {initial }} \sim 4 M_{\odot}$ (van Loon et al. 2000).

\subsubsection{IRAS $05329-6708$}

The brightest AGB star in the LMC at $25 \mu \mathrm{m}$, it was first identified with the optically visible semi-regular variable TRM060 (Reid et al. 1990) before the IRAS source was identified in the near-IR at $\sim 26^{\prime \prime} \mathrm{S}$ of TRM060 by
Wood et al. (1992). The region around IRAS 05329-6708 is very crowded, with a PN (TRM058 = LI-LMC1280) at $2.2^{\prime} \mathrm{SW}$, and an M4 Iab RSG (HV5933 = TRM063 = IRAS 05334-6706) at 4.2' NE. The latter has IRAS 12 and $25 \mu \mathrm{m}$ flux densities of 0.30 and $0.21 \mathrm{Jy}$, respectively.

$\mathrm{H}_{2} \mathrm{O}$ maser emission was tentatively detected (Fig. 6, top panel) at $22 \mathrm{GHz}$ at Parkes (1997 run), centred at $v_{\text {hel }}=347 \mathrm{~km} \mathrm{~s}^{-1}$. The peak has a FWHM of $\sim 5 \mathrm{~km} \mathrm{~s}^{-1}$. The integrated flux of the emission is $0.11 \mathrm{Jy} \mathrm{km} \mathrm{s}{ }^{-1}$, corresponding to a photon flux of $1.710^{44} \mathrm{~s}^{-1}$. It is not certain whether the $22 \mathrm{GHz}$ peak corresponds to the stellar velocity, or whether the $\mathrm{OH}$ and $\mathrm{H}_{2} \mathrm{O}$ masers refer to the same source. The $86 \mathrm{GHz} \mathrm{SiO}$ maser spectrum (Fig. 6, middle panel) is noisy, but there is positive signal (integrated flux $\sim 0.11 \mathrm{Jy} \mathrm{km} \mathrm{s}^{-1}$ ) within $1 \mathrm{~km} \mathrm{~s}^{-1}$ of the $\mathrm{H}_{2} \mathrm{O}$ maser peak, and some spikes within the velocity range of the $\mathrm{OH}$ emission.

\subsubsection{IRAS 05402-6956}

This star is $\sim 3^{\prime} \mathrm{W}$ of an $\mathrm{H}$ II region (DEM L275 \& 277).

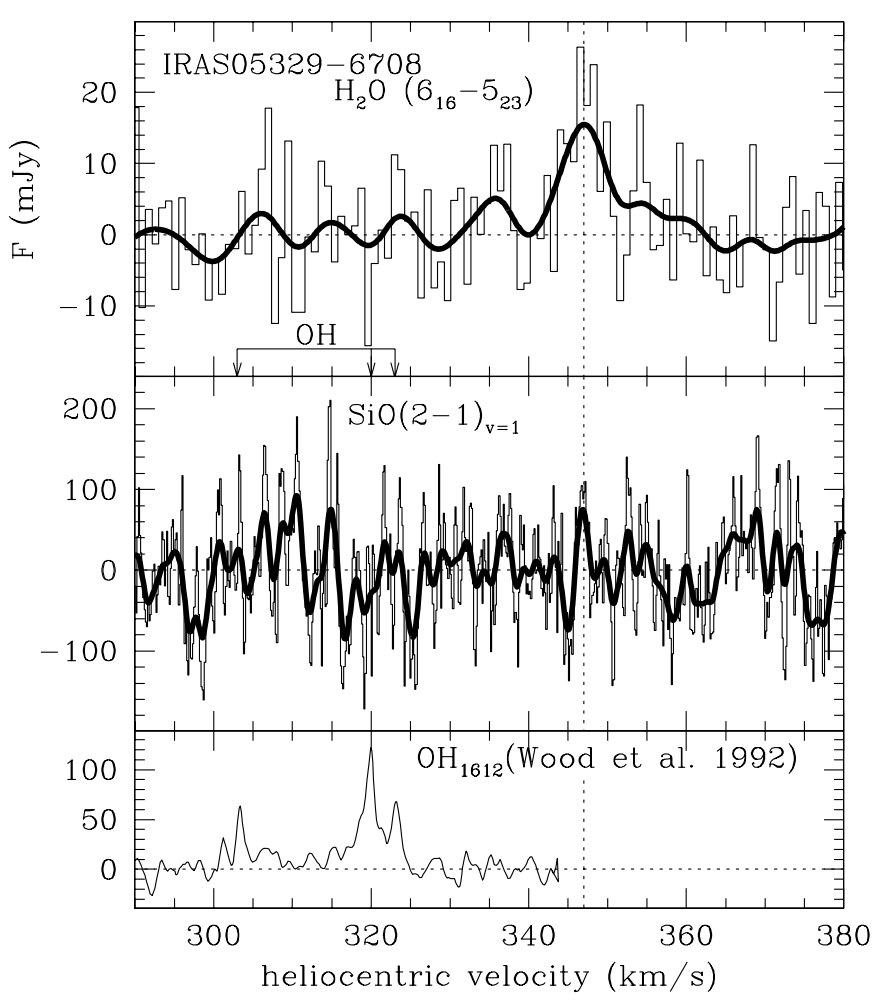

Fig. 6. IRAS 05329-6708: spectra of the tentative detections of $22 \mathrm{GHz} \mathrm{H}_{2} \mathrm{O}$ (top; Parkes 1997) and $86 \mathrm{GHz} \mathrm{SiO}$ (middle; SEST) maser emission. The boldfaced curves are the spectra smoothed by a Gaussian of $\sigma=2$ and $0.5 \mathrm{~km} \mathrm{~s}^{-1}$, respectively. The velocities are heliocentric. Also indicated are the velocities of the strongest $1612 \mathrm{MHz} \mathrm{OH}$ maser peaks (arrows), and heliocentric velocity $347 \mathrm{~km} \mathrm{~s}^{-1}$ (vertical dotted line). The $1612 \mathrm{MHz}$ spectrum of Wood et al. (1992) is plotted in the lower panel for comparison 


\section{4. $A G B$ stars in the $S M C$}

No $\mathrm{H}_{2} \mathrm{O}$ maser emission could be positively detected. To date, no circumstellar maser emission has been detected from any source in the SMC.

\subsubsection{IRAS 00483-7347}

This star is a late-M type LPV of $M_{\mathrm{bol}} \sim-7 \mathrm{mag}$ (Wood et al. 1992; Groenewegen \& Blommaert 1998). Castilho et al. (1998) measure some Li-enhancement. Their data suggest that the stellar photosphere is only mildly metalpoor - quite surprising for a star in the SMC. An H I shell (\#157 in Staveley-Smith et al. 1997) is located at $\sim 1.5^{\prime}$ and $v_{\text {hel }} \sim 156 \mathrm{~km} \mathrm{~s}^{-1}$.

A $3-\sigma$ peak $(\sim 150 \mathrm{mJy})$ at $v_{\text {hel }} \sim 153 \mathrm{~km} \mathrm{~s}^{-1}$ in the $22 \mathrm{GHz}$ spectrum with Mopra could not be confirmed with Parkes (Fig. 7). It would coincide with the maximum optical depth of the self-absorbed $21 \mathrm{~cm} \mathrm{H} \mathrm{I} \mathrm{(ATCA+Parkes)}$ at $v_{\text {hel }} \sim 157 \mathrm{~km} \mathrm{~s}^{-1}$ (Stanimirović et al. 1999).

\subsubsection{IRAS $00486-7308$}

This source is in the IRAS-FSC rather than the IRASPSC. The mid-IR flux densities were determined by Groenewegen \& Blommaert (1998). It coincides with the extended source IRAS 0048-731, which may be identified with the H II region LHA 115-N 36 and an H I shell (\#156 in Staveley-Smith et al. 1997). The most plausible counterpart of the IRAS point source is GM103, a luminous late-M type AGB star (Groenewegen \& Blommaert 1998) from which $10 \mu \mathrm{m}$ silicate dust emission was detected by Groenewegen et al. (1995). An emission-line star (\#368 in

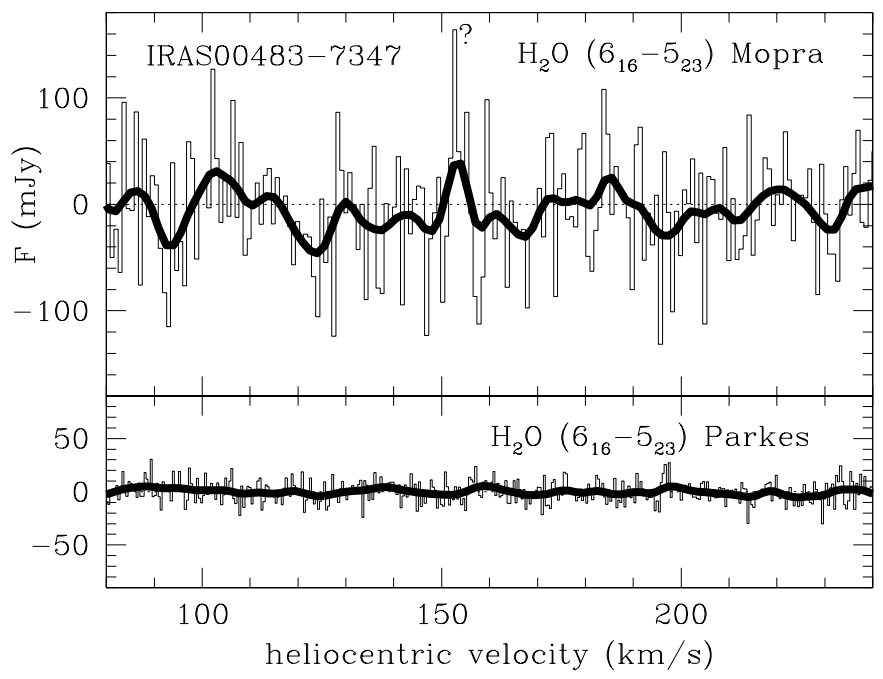

Fig. 7. IRAS 00483-7347: Mopra \& Parkes (2000) $22 \mathrm{GHz}$ spectra. The velocities are heliocentric. The boldfaced curves are the spectra smoothed by a Gaussian of $\sigma=2.0 \mathrm{~km} \mathrm{~s}^{-1}$. A possible $\mathrm{H}_{2} \mathrm{O}$ maser peak at $v_{\text {hel }} \sim 153 \mathrm{~km} \mathrm{~s}^{-1}$ in the Mopra spectrum could not be confirmed at Parkes despite the much lower noise levels
Meyssonnier \& Azzopardi 1993) and a carbon star (\#323 in Rebeirot et al. 1993) are $\sim 0.5^{\prime}$ away.

\subsubsection{IRAS $01074-7140$}

This is a luminous M5e-type Li-enhanced variable AGB star (Wood et al. 1983; Whitelock et al. 1989; Smith et al. 1995; Zijlstra et al. 1996; van Loon et al. 1998a; Groenewegen \& Blommaert 1998), located $\sim 0.8^{\prime}$ from the centre of an H I shell (\#369 in Staveley-Smith et al. 1997).

\section{5. $H$ II regions in the $L M C$}

No $43 \mathrm{GHz} \mathrm{SiO}$ masers were found in any of these objects, but at rather high detection thresholds. No other radio observations were made.

\subsubsection{IRAS 04491-6915}

This source is associated with the $\mathrm{H}$ II region DEM L2 (LHA 120-N77D).

\subsubsection{IRAS 04514-6931}

The extremely red mid-IR colour suggests that this is a (compact) H II region.

\subsubsection{IRAS 04546-6915}

This source is associated with the Wolf Rayet star HD 32014 in/and the cluster NGC 1748, situated in the complex H II region DEM L22.

\subsubsection{IRAS 04571-6627}

This source is located in the open cluster IC 2116 that contains other (early-type) evolved stars and that is embedded in the $\mathrm{H}$ II region LHA 120-N 11A (Parker et al. 1992; Rosado et al. 1996).

\subsubsection{IRAS 05198-6941}

This luminous IR object is in a region of high stellar and nebular density (LHA 120-N 120: Laval et al. 1992). Loup et al. (1997) identify the IRAS point source with the WC star HD 35517 (\#559 in Bohannan \& Epps 1974; see also Laval et al. 1994). Within $\sim 1^{\prime}$ are a multiple system (IDS 05201-6945) containing a B0 Iab supergiant, and an emission-line star (\#560 in Bohannan \& Epps 1974).

\subsubsection{IRAS 05325-6743}

This is associated with the $\mathrm{H}$ II region LHA 120-N 57A. 
Table 5. Kinematics for the presently known circumstellar masers in the LMC. Outflow and stellar velocities $\left(v_{\infty}\right.$ and $v_{\star}$, in $\mathrm{km} \mathrm{s}^{-1}$ ) are derived both from $\mathrm{OH}$ alone (half the separation of the main peaks in the blue- and red-shifted emission components) and from a combination of $\mathrm{OH}$ and $\mathrm{SiO}$ and/or $\mathrm{H}_{2} \mathrm{O}$ (the separation between the main peak of the blue-shifted $\mathrm{OH}$ emission and the central peak of the $\mathrm{SiO}$ and/or $\mathrm{H}_{2} \mathrm{O}$ emission). Uncertain values are within parentheses. Also given are the $21 \mathrm{~cm} \mathrm{H}$ I heliocentric velocities from the maps in LR-1992 $=$ Luks \& Rohlfs (1992)

\begin{tabular}{c|rr|rr|c}
\hline \hline IRAS-PSC & \multicolumn{2}{|c|}{$\mathrm{OH}$ alone } & \multicolumn{2}{|c|}{$\mathrm{OH}+\mathrm{SiO} / \mathrm{H}_{2} \mathrm{O}$} & \multicolumn{2}{c}{$\mathrm{LR}-1992$} \\
& $v_{\infty}$ & $v_{\star}$ & $v_{\infty}$ & $v_{\star}$ & $v_{\mathrm{HI}}$ \\
\hline $04407-7000$ & - & 239 & - & - & {$[250,255]$} \\
$04545-7000$ & $(8)$ & 266 & - & - & {$[245,250]$} \\
$04553-6825$ & 7 & 260 & 26 & 278 & {$[280,285]$} \\
$05216-6753$ & - & - & - & 284 & {$[295,300]$} \\
$05280-6910$ & 17 & 272 & 17 & 272 & {$[270,275]$} \\
$05298-6957$ & 11 & 282 & - & - & {$[265,270]$} \\
$05329-6708$ & 11 & 312 & $(44)$ & $(347)$ & {$[300,305]$} \\
$05402-6956$ & 11 & 272 & - & - & {$[260,265]$} \\
\hline
\end{tabular}

\section{Stellar and outflow velocities from circumstellar masers in the LMC}

The presently available data on the stellar and outflow velocities of $\mathrm{OH} / \mathrm{IR}$ stars in the MCs are summarised in Table 5. Literature values are largely based on the $\mathrm{OH}$ maser emission profile alone. The listed expansion velocities in that case are derived from the separation of the strongest peaks in the blue- and red-shifted emission components, respectively. Some authors use instead the maximum extension of the emission (rather than the peaks) as a measure for the expansion velocity (e.g. Zijlstra et al. 1996). Groenewegen et al. (1998) compare expansion velocities derived from the $\mathrm{OH}$ peaks with those derived from the width of the thermal CO emission and find that the former are on average smaller by a factor 1.12 (see also Lewis 1991). In some cases for which additional $\mathrm{SiO}$ and/or $\mathrm{H}_{2} \mathrm{O}$ maser emission was discovered, the $\mathrm{OH}$ masers appear to represent the part of the CSE in front of the star. Hence the outflow velocities may have been severely underestimated. Revised values are listed, using the $\mathrm{OH}$ peak velocities in relation to other maser peaks. The interpretation of the maser emission from IRAS 05329-6708, and hence its stellar and expansion velocities, is rather uncertain.

\subsection{Stellar velocities and local $H$ I velocities}

The velocities of the $\mathrm{OH} / \mathrm{IR}$ stars may be compared with the velocity of the local ISM. Luks \& Rohlfs (1992) present a low spatial resolution map of $21 \mathrm{~cm} \mathrm{H}$ I velocities for the LMC disk, from which approximate heliocentric velocities are listed in Table 5. Kim et al. (1999) present a high spatial resolution map of $21 \mathrm{~cm} \mathrm{H}$ I velocities, from which nine individual spectra centred on and around the position of each $\mathrm{OH} / \mathrm{IR}$ star are extracted and averaged (Fig. 8).
There is good agreement between the radio $\mathrm{H}$ I emission and the $\mathrm{H} \alpha$ emission around IRAS 04553-6825 (Fig. B.1). Both agree with its $\mathrm{SiO}$ and $\mathrm{H}_{2} \mathrm{O}$ maser velocities, as is to be expected for such a massive RSG. The $\mathrm{H}_{2} \mathrm{O}$ maser emission from IRAS $05216-6753$ provides for the first time a radial stellar velocity for this heavily obscured object, consistent with the local H I velocity. The $\mathrm{H}_{2} \mathrm{O}$ maser emission from IRAS 05280-6910 peaks at the $\mathrm{OH} 1612 \mathrm{MHz}$ centroid. Its stellar radial velocity deviates significantly from the bulk of the $\mathrm{H}$ I emission, with which the $\mathrm{OH} 1665 \mathrm{MHz}$ emission is associated. The $\mathrm{H}_{2} \mathrm{O}$ maser in IRAS 05329-6708 suggests an exceptionally high stellar velocity. It is seen projected on LMC4 (Meaburn 1980), a supergiant shell aged $\sim 10^{7} \mathrm{yr}$, and peculiar velocities are not surprising: high-velocity $\mathrm{H}_{2} \mathrm{O}$ masers are also found in 30 Doradus (van Loon \& Zijlstra 2000). Although most supergiants in the same (projected) region have stellar velocities similar to the centre velocity of the $\mathrm{OH}$ profile (Prévot et al. 1989), a few stars are found with radial velocities $\sim 350 \mathrm{~km} \mathrm{~s}^{-1}$ (Fehrenbach \& Duflot 1982).

Most OH/IR stars in the LMC follow the kinematics of the gas - in particular the AGB stars IRAS 04545-7000, IRAS 05298-6957 and IRAS 05402-6956. As the referee pointed out, this is remarkable because Galactic AGB stars show a large velocity spread. The observation that the velocities of the $\mathrm{OH} / \mathrm{IR}$ stars in the LMC do not generally deviate (much) from the motion of the gas may be due to the fact that the $\mathrm{OH} / \mathrm{IR}$ stars are relatively massive. Even the AGB stars amongst them probably have initial masses $M_{\text {initial }} \gtrsim 4 M_{\odot}$, otherwise they would have become carbon stars (Wood 1998; van Loon et al. 2000), and their ages are $t \lesssim 200 \mathrm{Myr}$. If they have formed in the disk of the LMC, they may still trace its rotation.

\subsection{Outflow kinematics in IRAS 04553-6825}

The $\mathrm{H}_{2} \mathrm{O}$ maser emission from IRAS 04553-6825 (Fig. 2), with its double blue-shifted emission peaks, reveals important information about the acceleration of the outflow. In first instance only the central main peak of $\mathrm{H}_{2} \mathrm{O}$ maser emission was discovered (van Loon et al. 1998b). Although most (circum-)stellar and maser properties of IRAS 04553-6825 are virtually identical to those of NML Cyg, a Galactic RSG (Morris \& Jura 1983; van Loon et al. 1998b), the $\mathrm{H}_{2} \mathrm{O}$ maser emission from NML Cyg (Richards et al. 1996) nearly entirely arises from a bright double peaked structure at blue-shifted velocities indicating outflow velocities in the $\mathrm{H}_{2} \mathrm{O}$ masing region of $\sim 15 \mathrm{~km} \mathrm{~s}^{-1}$ - a main peak of $\mathrm{H}_{2} \mathrm{O}$ maser emission centred at the stellar velocity has only occasionally been reported for NML Cyg. Comparison with its $\mathrm{OH}$ maser emission indicative of outflow velocities of $\sim 27 \mathrm{~km} \mathrm{~s}^{-1}$ led to the interpretation of the $\mathrm{H}_{2} \mathrm{O}$ masing region in NML Cyg to be located in the accelerating part of the outflow where the matter has not yet reached the local escape velocity (Richards et al. 1996). The detection of similar blueshifted $\mathrm{H}_{2} \mathrm{O}$ maser emission from IRAS 04553-6825 now 


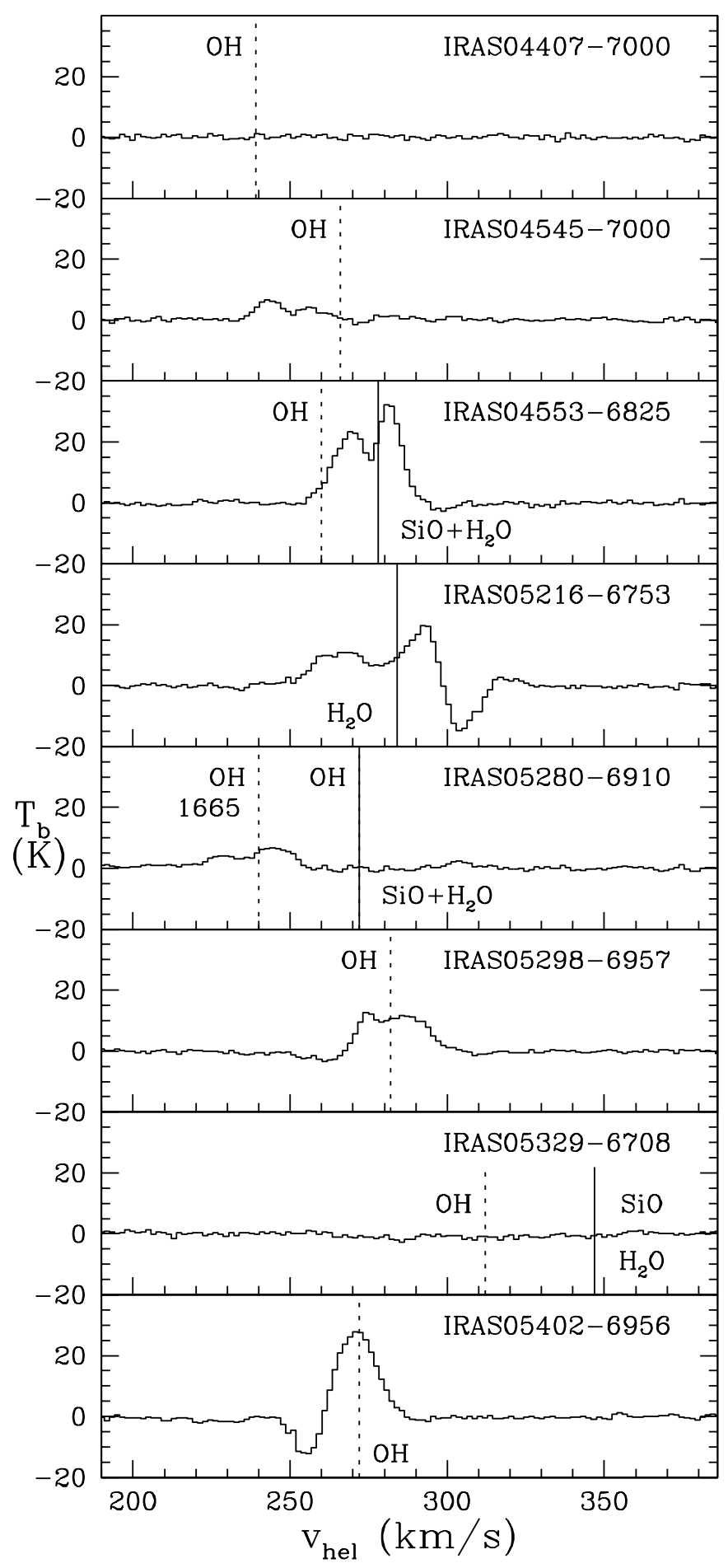

Fig. 8. $21 \mathrm{~cm} \mathrm{H}$ I spectra, constructed by averaging nine spectra from Kim et al. (1999) centred on and around the position of each $\mathrm{OH} / \mathrm{IR}$ star. The stellar velocities are indicated as a vertical line (solid: $\mathrm{SiO}$ and/or $\mathrm{H}_{2} \mathrm{O}$; dotted: $1612 \mathrm{MHz} \mathrm{OH}$ )

for the first time also allows us to measure the acceleration of the outflow for this metal-poor RSG in the LMC.

The main $\mathrm{H}_{2} \mathrm{O}$ maser peak in IRAS 04553-6825 was interpreted by van Loon et al. (1998b) either (i) to be radially beamed and hence indicate very low outflow velocities of $\sim 1 \mathrm{~km} \mathrm{~s}^{-1}$ in a dust-free inner CSE, or (ii) to be tangentially beamed and centred at the stellar velocity. The detection of the blue-shifted $\mathrm{H}_{2} \mathrm{O}$ maser emission now strongly favours the latter. The blue-shifted maser peaks suggest the emission is radially beamed and thus measures the radial outflow velocity of the material expelled from the star, in the region where the dust formation is thought to take place. Material is accelerated from $18 \mathrm{~km} \mathrm{~s}^{-1}$ in the $\mathrm{H}_{2} \mathrm{O}$ masing zone to $26 \mathrm{~km} \mathrm{~s}^{-1}$ in the $\mathrm{OH}$ masing zone. The duplicity of both the $\mathrm{H}_{2} \mathrm{O}$ and $\mathrm{OH}$ blueshifted emission suggests a second kinematic component in the CSE, accelerating from $13\left(\mathrm{H}_{2} \mathrm{O}\right)$ to $14(\mathrm{OH}) \mathrm{kms}^{-1}$. This slower component may be closer to the star as suggested by the relatively stronger mainline $\mathrm{OH} 1665 \mathrm{MHz}$ maser emission from that component (Wood et al. 1992). It is remarkable that the $\mathrm{OH} 1665 \mathrm{MHz}$ emission profile is broader than that of the $\mathrm{OH} 1612$ emission, a phenomenon that is attributed to either (or a combination of) Zeeman broadening, clumpiness, velocity fluctuations or axi-symmetric winds (Sivagnanam \& David 1999).

The kinematic data for the CSE of IRAS 04553-6825 are very similar to the kinematics in the CSE of NML Cyg, suggesting that the outflow kinematics including the acceleration mechanism does not depend on metallicity. Other data, however, seem to support theoretical expectations of lower velocities in CSEs of lower metallicity (van Loon 2000). One way to reconcile both views is if the distance to NML Cyg were $\sim 1.3 \mathrm{kpc}$ rather than $2 \mathrm{kpc}$ : the outflow velocity scales with metallicity $Z$ and luminosity $L$ as $v_{\infty} \propto \sqrt{Z} \sqrt[4]{L}$ (van Loon 2000) with $Z \sim 0.02$ for NML Cyg (solar) and $Z \sim 0.008$ for IRAS 04553-6825 (van Loon et al. 1998b and Appendix B).

The velocity range over which $\mathrm{SiO}$ maser emission is seen from IRAS 04553-6825 covers the entire velocity range of the $\mathrm{H}_{2} \mathrm{O}$ maser emission. If the strong double peak of $\mathrm{SiO}$ maser emission is tangentially beamed then the velocity separation might imply a rotational velocity component of the inner CSE. If the double $\mathrm{SiO}$ peak is radially beamed, however, then it suggests moderate outflow velocities in the dust-free inner CSE of $\sim 4 \mathrm{~km} \mathrm{~s}^{-1}$. In any case, the simultaneous presence of $\mathrm{SiO}$ emission over a large velocity extent and strong discrete peaks of $\mathrm{SiO}$ emission indicate that the velocity field of the inner CSE is highly complex.

Results from echelle spectroscopic observations of IRAS $04553-6825$ in the 0.6 to $0.9 \mu \mathrm{m}$ region that generally support the kinematic picture for the CSE of this star are described in Appendix B.

\subsection{Outflow kinematics in IRAS 05280-6910}

The $\mathrm{H}_{2} \mathrm{O}$ maser emission from IRAS 05280-6910 essentially shows the same features as seen in IRAS 04553-6825, and thus carries the same potential for analysing the kinematics of its CSE: the narrow main peak is interpreted as tangentially beamed radiation centred at the stellar velocity, and the blue-shifted emission (in the case of IRAS 05280-6910 probably its 
red-shifted counterpart is seen as well) can be explained as radially beamed radiation indicating the radial outflow velocity in the $\mathrm{H}_{2} \mathrm{O}$ masing region of the CSE close to the region of dust formation. The outflow in the CSE of IRAS $05280-6910$ is being accelerated from $\sim 6 \mathrm{~km} \mathrm{~s}^{-1}$ in the $\mathrm{H}_{2} \mathrm{O}$ masing region to $\sim 17 \mathrm{~km} \mathrm{~s}^{-1}$ in the outer CSE from where the $\mathrm{OH} 1612 \mathrm{MHz}$ maser emission arises.

Double-peaked red-shifted $\mathrm{SiO}$ emission may have been detected, with equal velocity separation but slightly larger receding velocities than the red-shifted $\mathrm{OH}$ emission. This suggests that material in the dust-free inner CSE may exhibit a wide range of velocities, possibly exceeding the final wind velocity (Cernicharo et al. 1997).

\subsection{Outflow kinematics of Magellanic and Galactic circumstellar masers}

Expansion velocities from the separation of the two $\mathrm{OH}$ peaks versus maximum peak flux density are plotted in Fig. 9 for the $\mathrm{OH} / \mathrm{IR}$ stars in the Galactic Centre from Lindqvist et al. (1992a) and Sjouwerman et al. (1998), after scaling their flux densities from the distance of the Galactic Centre to the distance of the LMC. Also plotted are the expansion velocities for the $\mathrm{OH} / \mathrm{IR}$ stars in the LMC, as derived from the combination of all detected maser peaks. Expansion velocities tend to be below average for the brightest $\mathrm{OH}$ sources. For the LMC sources the statistics are very poor, with only one well-determined expansion velocity for an AGB star (IRAS 05298-6957) suggestive of smaller expansion velocities at lower metallicity (see also Wood et al. 1992; Zijlstra et al. 1996; van Loon 2000). The new maser data presented here for IRAS 05329-6708, however, suggest that this source may exhibit an exceptionally large expansion velocity even when compared to the expansion velocities of Galactic post-AGB objects (Zijlstra et al. 2000). Disregarding IRAS $04553-6825$ and IRAS $05329-6708$, the average expansion velocity of the remaining four LMC objects is $v \sim$ $12 \pm 3 \mathrm{~km} \mathrm{~s}^{-1}$, which may be compared to $v \sim 14 \pm 2 \mathrm{~km} \mathrm{~s}^{-1}$ of the six brightest $\mathrm{OH}$ masers in the Galactic Centre. Hence the expansion velocity of bright $\mathrm{OH}$ sources in the LMC is $\sim 20 \%$ lower than that of similarly bright $\mathrm{OH}$ masers in the Galactic Centre, but the data is also consistent with no difference in expansion velocity.

In the Galactic Centre, unlike the $13 \mathrm{SiO}$ masers (Lindqvist et al. 1991) that peak within a few $\mathrm{km} \mathrm{s}^{-1}$ of the mid-velocity of the $\mathrm{OH}$ peaks - i.e. centred at the stellar velocity - the $\mathrm{H}_{2} \mathrm{O}$ detections (Lindqvist et al. 1990) peak at or very near the $\mathrm{OH}$ peak velocities. This suggests that the wind has already (nearly) reached its terminal velocity before leaving the $\mathrm{H}_{2} \mathrm{O}$ masing zone. In three out of four cases the blue-shifted $\mathrm{H}_{2} \mathrm{O}$ peak is (much) brighter than the red-shifted peak. $\mathrm{H}_{2} \mathrm{O}$ masers in the LMC, however, peak at the stellar velocity indicated by the centroid of the $\mathrm{SiO}$ maser emission, rather than at (one of) the $\mathrm{OH}$ maser peak(s). Secondary $\mathrm{H}_{2} \mathrm{O}$ peaks suggest that the wind must still experience substantial acceleration af-

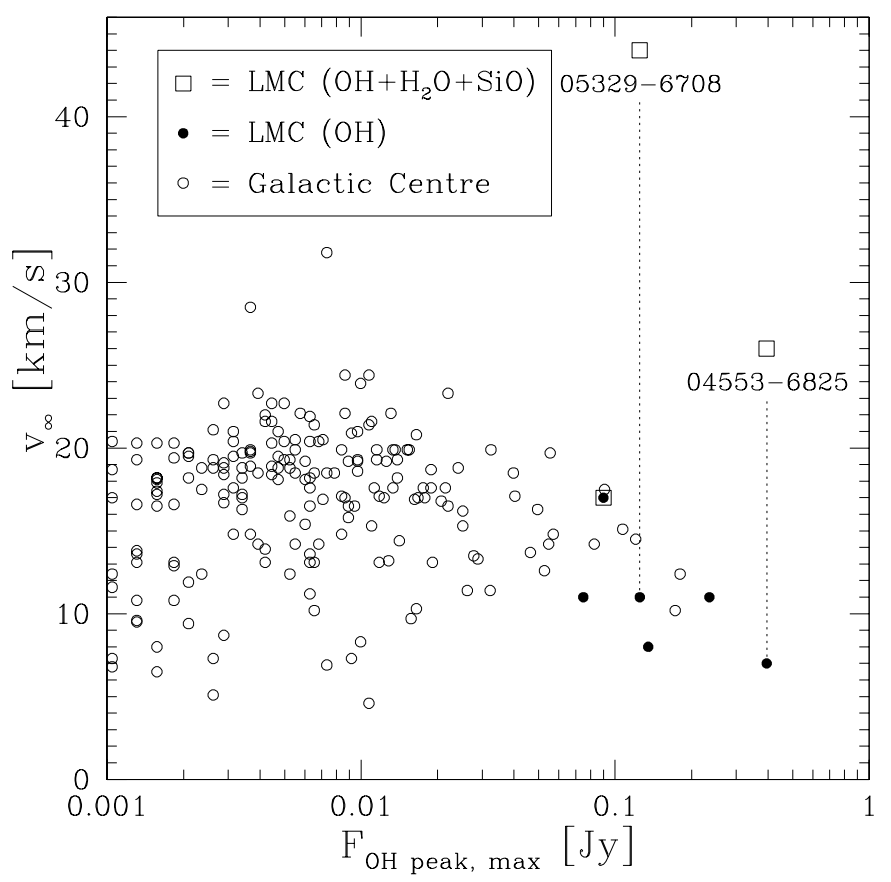

Fig. 9. Expansion velocities of the circumstellar shells derived from the separation of the $\mathrm{OH}$ maser peaks, versus the flux density of the brightest $\mathrm{OH}$ maser peak (at the LMC distance). Expansion velocities derived from the combination of all detected masers are given too for the LMC sources (squares)

ter leaving the $\mathrm{H}_{2} \mathrm{O}$ masing zone. This may be understood by a less efficient acceleration in the $\mathrm{H}_{2} \mathrm{O}$ masing zone of low-metallicity CSEs, which results in a stellar wind that is still being accelerated upon entering the $\mathrm{OH}$ masing zone. This could cause multiple, thin $\mathrm{OH}$ masing shells to give rise to the rather irregular $\mathrm{OH}$ emission profiles as observed in the LMC.

\subsection{Asymmetric emission profiles: Non-spherical outflow or radiation transfer effects?}

All masers observed in the LMC are blue-asymmetric in the sense that the masing material approaching Earth (after correcting for the stellar velocity with respect to Earth) appears brighter than the receding matter (Fig. 10): the $\mathrm{OH}, \mathrm{H}_{2} \mathrm{O}$ and $\mathrm{SiO}$ maser emission from IRAS 04553-6825, the $\mathrm{OH}$ and $\mathrm{H}_{2} \mathrm{O}$ maser emission from IRAS $05280-6910$, the $\mathrm{OH}$ maser emission from all four AGB stars detected by Wood et al. (1992) - if interpreting IRAS 05329-6708 in combination with the tentative $\mathrm{H}_{2} \mathrm{O}$ maser detection - and possibly also the single-peaked $\mathrm{OH}$ maser emission from IRAS $04407-7000$. Does this reflect outflow complexity (Zijlstra et al. 2000), or is it merely due to the origin and propagation of the amplified radiation field? Blue-asymmetric emission profiles (Norris et al. 1984; Sivagnanam et al. 1990) arise if the (radially-beamed) masers amplify stellar light or free-free emission from the inner part of the CSE, rather than radiation from the dusty CSE, and/or the receding maser spots are occulted by the star or an optically thick free-electron reservoir. 


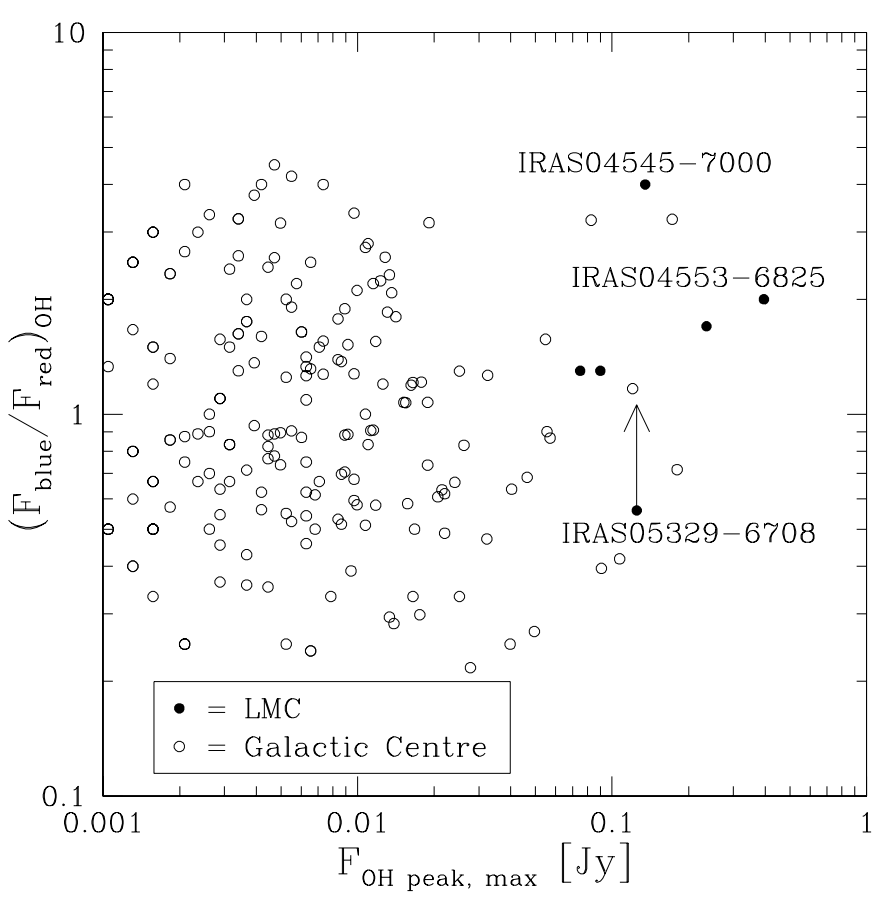

Fig. 10. Ratio of the flux densities of the blue- and red-shifted $\mathrm{OH}$ maser peaks, versus the flux density of the brightest $\mathrm{OH}$ maser peak (at the LMC distance)

The blue asymmetry is expected to be more pronounced in RSGs such as IRAS 04553-6825 than in AGB stars such as IRAS 05298-6957, because (i) at mm wavelengths, the ratio of stellar light to CSE radiation is larger for RSGs than for more compact AGB stars that have optically thicker CSEs (van Loon 2000), and (ii) RSGs are generally warmer than AGB stars and hence the freeelectron abundance is higher around RSGs. The blue asymmetry may be more pronounced at lower metallicity, because (i) at $\mathrm{mm}$ wavelengths, the ratio of stellar light to CSE radiation is smaller because of the lower dust-to-gas ratio, and (ii) the free-electron abundance is higher due to the warmer photospheres of low-metallicity stars.

\section{Photon fluxes of Magellanic and Galactic circumstellar masers}

\subsection{OH masers}

The $\mathrm{OH}$ masers in the LMC have large photon fluxes compared to what is typical for the Galactic Centre (see Fig. 9), where the $\mathrm{OH}$ luminosity function peaks at $2.510^{43} \mathrm{~s}^{-1}$ (Sjouwerman et al. 1998). The $\mathrm{OH}$ maser emission from IRAS $04553-6825$ is $~ 100$ times brighter (and so is NML Cyg, a very similar RSG in the Milky Way). The sixth brightest maser in the Galactic Centre is equally bright as the sixth brightest maser in the LMC, which suggests that a similar number of intermediate-age stars exist in the Galactic Centre and in the LMC. Saturated $\mathrm{OH}$ masers have flux densities $S_{\mathrm{OH}} \sim \frac{1}{4} \times S_{35 \mu \mathrm{m}}$. Although the detected $\mathrm{OH}$ masers in the Magellanic AGB stars meet the saturation criterion within a factor $\sim 2$, the detected $\mathrm{OH}$ masers in the two Magellanic RSGs are an order of magnitude below the saturation level.

The deepest search for $\mathrm{OH}$ sources in the LMC has been performed with a detection limit of $\sim 50 \mathrm{mJy}$, and several single-peaked sources were found, including IRAS 04407-7000 (van Loon et al. 1998a). A flux density of $50 \mathrm{mJy}$ from the LMC corresponds to $2 \mathrm{Jy}$ from the Galactic Centre. Of the 134 double and 16 single-peaked $\mathrm{OH}$ sources in Lindqvist et al. (1992a), only 12 have maximum flux densities $>2$ Jy. Out of these 12 sources, 8 would appear as a single peak. Hence, when searching for $\mathrm{OH}$ masers close to the detection limit, one is likely to find more single than double-peaked sources.

Many of the $1612 \mathrm{MHz}$ peaks of van Loon et al. (1998a) could not be identified with IR-bright point sources associated with CSEs around evolved stars. Interestingly, none of the Galactic Centre $\mathrm{OH}$ sources with maximum flux densities $>2$ Jy were recovered in the near-IR by Wood et al. (1998), who did identify a number of the fainter Galactic Centre $\mathrm{OH}$ sources with near-IR objects. Only two of these bright $\mathrm{OH}$ sources are identified with IRAS point sources (Appendix C).

Amongst the LPVs with known pulsation periods and bolometric magnitudes from Wood et al. (1998) the fraction of stars with detected $\mathrm{OH}$ emission incre0ases with redder $(K-L)_{0}: 0.22,0.77,0.90,0.95$ and 1.00 for colour bins $(\leftarrow, 1],[1,2],[2,3],[3,4]$ and $[4, \rightarrow)$ mag, respectively. No $\mathrm{OH}$ masers are detected from LPVs with $P \lesssim 500 \mathrm{~d}$. The $\mathrm{OH} / \mathrm{IR}$ stars in the LMC all have periods $900 \lesssim P \lesssim$ $1300 \mathrm{~d}$, but their colours are with $(K-L)_{0} \sim 2$ mag not extremely red (Trams et al. 1999) due to the low dust content as a result of a low metallicity.

\section{2. $\mathrm{H}_{2} \mathrm{O}$ masers}

Lindqvist et al. (1990) detected $22 \mathrm{GHz} \mathrm{H}_{2} \mathrm{O}$ maser emission from 4 out of $33 \mathrm{OH}$ sources in the Galactic Centre. Their detections typically have peak flux densities of 0.5 to $1 \mathrm{Jy}$, corresponding to $\sim 20 \mathrm{mJy}$ if the sources were at the distance of the LMC. The 3- $\sigma$ upper limits for their non-detections correspond to $\sim 10 \mathrm{mJy}$ at the distance of the LMC, which is comparable to the sensitivity of our search for $\mathrm{H}_{2} \mathrm{O}$ maser emission from Magellanic sources. Of the 13 Magellanic IRAS sources that were observed at $22 \mathrm{GHz}$, four were detected. Three of the IRAS-PSC identifications with $\mathrm{OH}$ masers in the Galactic Centre (Table C.1) were observed at $22 \mathrm{GHz}$ : OH359.675+0.069 and OH359.946-0.047 were detected (both identified with near-IR LPVs), but the bright $\mathrm{OH}$ maser OH359.762+0.120 (for which no near-IR counterpart has been identified) was not detected. Lewis (1998) already remarked that $22 \mathrm{GHz}$ detection rates are always $<100 \%$ (namely, 80\%), and even some bright $\mathrm{OH}$ sources are undetected at $22 \mathrm{GHz}$. He suggests that this may be due to the absence of density enhancements ("blobs") or an unfavourable orientation of a bipolar geometry. 
Hence, not all Magellanic OH/IR stars should be expected to exhibit (strong) $\mathrm{H}_{2} \mathrm{O}$ masers. Significant mass loss is required for the presence of circumstellar $\mathrm{H}_{2} \mathrm{O}$ masers, though: AGB stars with modest mass loss are far below the sensitivity of both the Magellanic and Galactic Centre $22 \mathrm{GHz}$ surveys (see Appendix A).

The ratio of $\mathrm{H}_{2} \mathrm{O}$ and $\mathrm{OH}$ photon fluxes is $\sim 0.1$ to 1 , and the ratio of $\mathrm{H}_{2} \mathrm{O}$ and $\mathrm{OH}$ peak flux densities is $\sim 1$, both for the Galactic Centre as well as the LMC masers. At lower metallicities the CSEs are optically thinner and provide less self-shielding from the interstellar UV radiation field, and hence $\mathrm{H}_{2} \mathrm{O}$ may be expected to be dissociated over a larger extent of CSEs in the LMC compared to those in the Galactic Centre (Huggins \& Glassgold 1982), thus yielding relatively faint $\mathrm{H}_{2} \mathrm{O}$ maser emission compared to the $\mathrm{OH}$ maser emission. The fact that no difference is seen between these relative intensities in the LMC and the Galactic Centre suggests that the effect of stronger dissociation might be cancelled by the effect of longer coherent paths throughout the more slowly accelerating wind in lower metallicity CSEs.

\subsection{SiO masers}

Lindqvist et al. (1991) detected $43 \mathrm{GHz} \mathrm{SiO}$ maser emission from 13 out of $31 \mathrm{OH}$ sources in the Galactic Centre, peaking at $S_{43} \sim 1 \mathrm{Jy}(\sim 30 \mathrm{mJy}$ at the LMC). Upper limits for the photon rates of Galactic non-detections at $43 \mathrm{GHz}$ were a few $10^{44} \mathrm{~s}^{-1}$. The upper limits for LMC sources using the Parkes dish are a few dozen times brighter and thus not very useful. None of the Galactic Centre $43 \mathrm{GHz}$ masers could be detected at $86 \mathrm{GHz}$ down to typical photon rates $\sim 10^{44} \mathrm{~s}^{-1}$. This is similar to the SEST limits for LMC sources and thus explains the difficulty in detecting $86 \mathrm{GHz}$ masers in the LMC.

Two of the Galactic Centre $\mathrm{OH}$ masers with IRAS-PSC identifications (Table C.1) were observed at $43 \mathrm{GHz}$, and both were detected (OH359.675+0.069 and $\mathrm{OH} 359.762+0.120)$. Three Galactic Centre $\mathrm{H}_{2} \mathrm{O}$ masers were observed at $43 \mathrm{GHz}$, of which two were detected (including OH359.675+0.069). In the LMC too, the brightest (circumstellar) $\mathrm{H}_{2} \mathrm{O}$ masers are also the brightest $\mathrm{SiO}$ masers. However, some quite bright mid-IR objects could not be detected in any maser transition - e.g. IRAS $05346-6949$.

$\mathrm{SiO}$ maser emission may be detected from IR objects with pulsation periods $P \gtrsim 400 \mathrm{~d}$, and it is always present when $P \gtrsim 800 \mathrm{~d}$ (Fig. 11). The OH/IR stars in the LMC all have $P \gtrsim 900 \mathrm{~d}$ and are thus expected to exhibit $\mathrm{SiO}$ maser emission, probably not much below the sensitivity limits of our searches. With $(K-L)_{0} \sim 2 \mathrm{mag}$ their $86 \mathrm{GHz} \mathrm{SiO}$ maser emission is expected to be similarly bright as their $43 \mathrm{GHz}$ masers (Nyman et al. 1993). Lindqvist et al. (1991) find that for Miras - probably the progenitors of $\mathrm{OH} / \mathrm{IR}$ stars - the $\mathrm{SiO}$ masers can often be $10^{2}$ times as intense as the $\mathrm{OH}$ masers. $\mathrm{OH}$ luminosities scale with $35 \mu \mathrm{m}$ luminosities, and $\mathrm{OH}$ outflow velocities

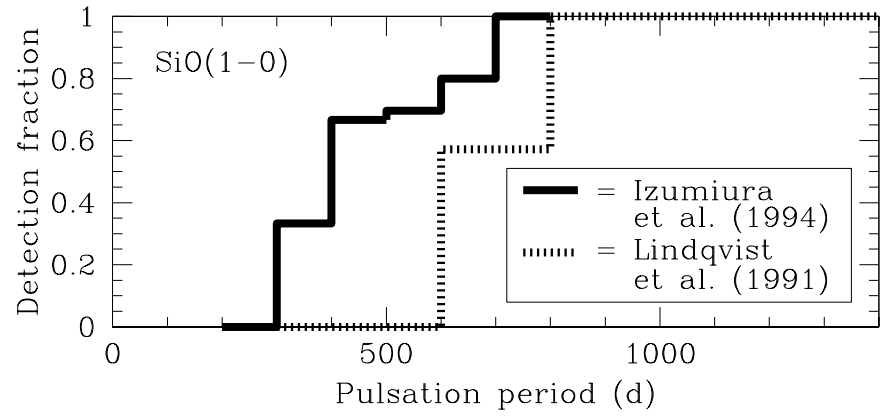

Fig. 11. Detection fraction for $\mathrm{SiO} J=1 \rightarrow 0$ masers versus pulsation period, for the Galactic Bulge (solid: Izumiura et al. 1994) and the Galactic Centre (dotted: Lindqvist et al. 1991)

reach a maximum for OH/IR stars (Sivagnanam et al. 1989), indicating that $\mathrm{OH} / \mathrm{IR}$ stars experience stronger mass outflows than Miras. The ratio of $\mathrm{SiO}$ and $\mathrm{OH}$ photon fluxes (or peak flux densities) is about unity both for the Galactic Centre and LMC sources, which suggests that also in the LMC the brightest $\mathrm{OH}$ masers are already experiencing heavy mass loss for some time.

Alcolea et al. (1990) found a clear correlation between the visual amplitude of pulsation and the pumping efficiency of the $\mathrm{SiO}$ masers. For our obscured LMC sources no visual photometric monitoring data is available. It is known, however, that the $\mathrm{SiO}$ maser emission correlates with the IR lightcurve (Nyman \& Olofsson 1986; Alcolea et al. 1999), and hence we investigate here whether the $\mathrm{SiO}$ maser intensity correlates with the IR amplitude. In Table 6 IR photometric variability data is summarised for relatively nearby stars in the Milky Way that were monitored by Harvey et al. (1974) and Le Bertre (1993). The latter source, if available, is preferred because it is more modern by nearly two decades. Where only narrow-band $N_{1,2,3}$ was obtained the broad-band $N$ is approximated by an unweighted average. $\operatorname{SiO}_{v=1}(J=2 \rightarrow 1)$ peak flux densities were compiled from recent literature and averaged if more data was available. For the LMC sources similar data is compiled, but at $10 \mu \mathrm{m}$ too few epochs of measurements exist to allow an estimate of the amplitude.

The flux density ratio of the peak of the $\operatorname{SiO}_{v=1}(J=$ $2 \rightarrow 1)$ and the stellar flux at $2.2 \mu \mathrm{m}$ increases for larger $2.2 \mu \mathrm{m}$ amplitudes (Fig. 12). This means that larger amplitudes cause relatively stronger maser pumping, probably because of the relatively stronger shocks travelling through the inner dust-free part of the CSE. A similar correlation is found for other near-IR amplitudes and for integrated fluxes of the $\mathrm{SiO}$ maser emission, but their lower accuracy leads to more scatter. The one secure and two tentative detections of $\mathrm{SiO}_{v=1}(J=2 \rightarrow 1)$ maser emission in the LMC, as well as the three useful upper limits are all consistent with $\mathrm{SiO}_{v=1}(J=2 \rightarrow 1)$ masers that are equally strong in the LMC and the Milky Way. Figure 12 suggests $\Delta K \sim 1$ to 2 mag for IRAS 05280-6910, but the DENIS $K$-band magnitude is with 8.195 (Cioni et al. 2000) very similar to the photometry of Wood et al. (1992). 
Table 6. IR magnitudes and their variability for LMC masers (Elias et al. 1986; Wood et al. 1992; Zijlstra et al. 1996; van Loon et al. 1998a) and Galactic masers (Harvey et al. 1974; Le Bertre 1993), and $\mathrm{SiO}_{v=1}(J=2 \rightarrow 1)$ peak flux densities (in Jy) for LMC masers (this work) and Galactic masers (Nyman \& Olofsson 1985; Alcolea et al. 1990; Haikala 1990; Le Bertre \& Nyman 1990; Nyman et al. 1993; Haikala et al. 1994; Bujarrabal et al. 1996; Cernicharo et al. 1997; GonzálezAlfonso et al. 1998; Herpin et al. 1998; Nyman et al. 1998)

\begin{tabular}{|c|c|c|c|c|c|}
\hline Star & $K$ & $\Delta K$ & $N$ & $\Delta N$ & $\mathrm{SiO}$ \\
\hline \multicolumn{6}{|l|}{ LMC masers } \\
\hline IRAS 04407-7000 & 9.05 & 1.40 & 5.03 & & $<0.35$ \\
\hline IRAS $04553-6825$ & 6.99 & 0.30 & 1.74 & & 0.15 \\
\hline IRAS 05216-6753 & 10.38 & 0.20 & 2.43 & & $<0.28$ \\
\hline IRAS $05280-6910$ & 8.19 & & 2.41 & & $(0.30)$ \\
\hline IRAS 05298-6957 & 10.29 & 2.00 & 4.14 & & $<0.29$ \\
\hline IRAS 05329-6708 & 9.87 & 1.90 & 3.81 & & $(0.12)$ \\
\hline \multicolumn{6}{|l|}{ Milky Way masers } \\
\hline IRAS 00193-4033 & 3.28 & 1.30 & -1.61 & 1.04 & 23 \\
\hline IRAS $22231-4529$ & 3.11 & 1.09 & -0.55 & 0.82 & 12 \\
\hline IRC-30100 & 1.79 & 0.27 & & & 34 \\
\hline IRC -30050 & 2.55 & 0.67 & & & 11 \\
\hline IRC-20540 & 2.21 & 0.99 & & & 10 \\
\hline IRC-20197 & 2.49 & 1.26 & -2.42 & 1.30 & 45 \\
\hline IRC-10529 & 2.33 & 1.50 & -3.31 & 2.85 & 53 \\
\hline IRC+10011 & 2.47 & 1.79 & -3.00 & 1.70 & 66 \\
\hline IRC +10523 & 1.85 & 1.07 & & & 10 \\
\hline NML Cyg & 0.60 & 0.51 & -5.30 & 0.51 & 42 \\
\hline NML Tau & -0.68 & 1.09 & & & 350 \\
\hline OH $02.60-0.4$ & 3.42 & 1.52 & & & 27 \\
\hline OH $26.5+0.6$ & 8.55 & 3.27 & & & 5 \\
\hline OH $285.05+0.07$ & 5.32 & 1.50 & -0.12 & 1.27 & 4 \\
\hline $\mathrm{OH} 286.50+0.06$ & 5.27 & 1.41 & -0.43 & 1.27 & 12 \\
\hline $\mathrm{OH} 300.93-0.03$ & 4.95 & 1.20 & & & 2 \\
\hline $\mathrm{OH} 315.22+0.01$ & 5.87 & 1.35 & & & 4 \\
\hline OH $341.12-0.01$ & 6.04 & 1.16 & & & 2 \\
\hline $\mathrm{OH} 342.01+0.25$ & 3.90 & 1.21 & & & 11 \\
\hline OH $344.83-1.67$ & 7.02 & 1.28 & & & 2 \\
\hline OH $346.86-0.18$ & 4.01 & 1.53 & & & 3 \\
\hline $\mathrm{OH} 349.18+0.20$ & 8.34 & 1.64 & & & 2 \\
\hline OH $358.16+0.50$ & 3.08 & 1.88 & & & 10 \\
\hline R Aql & -0.70 & 0.58 & -2.40 & 0.51 & 37 \\
\hline R Aqr & -1.09 & 0.81 & -3.58 & 0.70 & 140 \\
\hline RR Aql & 0.50 & 1.12 & -2.40 & 1.08 & 29 \\
\hline $\mathrm{S}$ Col & 1.63 & 0.57 & 0.02 & 0.29 & 3 \\
\hline $\mathrm{S} \mathrm{CrB}$ & 0.10 & 0.81 & -2.50 & 0.58 & 100 \\
\hline U Her & -0.20 & 0.81 & -2.50 & 0.51 & 140 \\
\hline U Ori & -0.60 & 0.90 & -2.70 & 0.58 & 110 \\
\hline VX Sgr & 0.20 & 1.30 & -4.20 & 0.90 & 280 \\
\hline VY CMa & -0.70 & 0.15 & -5.90 & 0.15 & 1700 \\
\hline W Hya & -3.10 & 0.51 & -5.00 & 0.28 & 680 \\
\hline
\end{tabular}

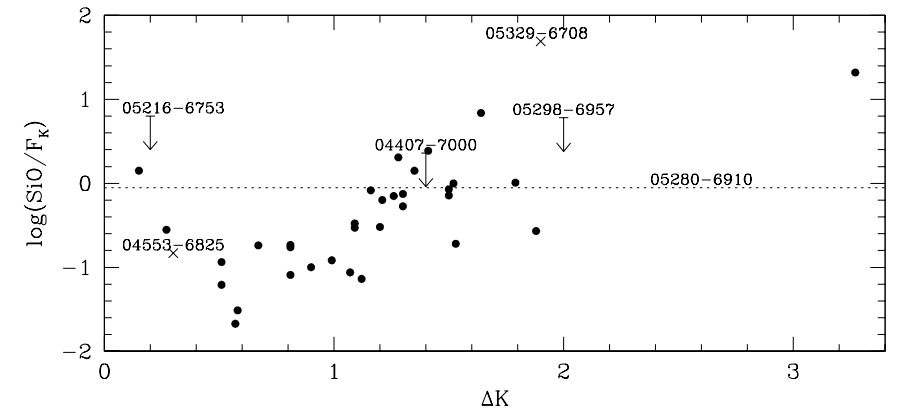

Fig. 12. Flux density ratio of $\operatorname{SiO}_{v=1}(J=2 \rightarrow 1)$ peak and $K$ band, versus $K$-band amplitude for Galactic (solid dots) and LMC masers from Table 6

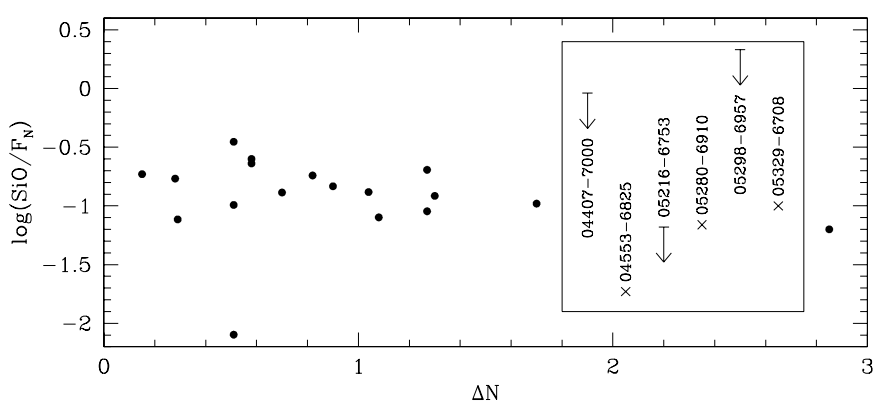

Fig. 13. Same as Fig. 12, but for the $N$-band

Interestingly, the flux density ratio of the peak of the $\mathrm{SiO}_{v=1}(J=2 \rightarrow 1)$ and the stellar flux at $10 \mu \mathrm{m}$ ( $N$-band $)$ is virtually independent of any near-IR amplitude (Fig. 13; plotted against the amplitude at $10 \mu \mathrm{m}$ ), and shows rather little scatter. This indicates a close connection between the shocks that pump the $\mathrm{SiO}$ maser, and the dust formation. It also suggests that both the SiO luminosity and the dust heating have a common origin, namely the stellar flux. Again, the LMC data (no $10 \mu \mathrm{m}$ amplitudes available) is roughly compatible with the same trend. Thus there is no strong evidence for a difference in maser strength at LMC metallicities compared to $\sim$ solar metallicity.

\section{Summary}

A five year effort to search for $\mathrm{SiO}$ and $\mathrm{H}_{2} \mathrm{O}$ maser emission from circumstellar envelopes in the LMC resulted in: - The secure detection of $86 \mathrm{GHz}^{\mathrm{SiO}}{ }_{v=1}(J=2 \rightarrow 1)$ and $22 \mathrm{GHz} \mathrm{H}_{2} \mathrm{O} 6_{16} \rightarrow 5_{23}$ maser emission from the luminous red supergiant IRAS 04553-6825. The average $\mathrm{SiO}$ spectrum of 65 hours worth of monitoring data shows unprecedented kinematic detail, probably indicating outflow and/or turbulent motions with $v_{\mathrm{SiO}} \sim$ a few $\mathrm{kms}^{-1}$ up to $\sim 18 \mathrm{~km} \mathrm{~s}^{-1}$ with respect to the stellar velocity. A new, improved signal-to-noise $\mathrm{H}_{2} \mathrm{O}$ spectrum also shows emission components at $v_{\mathrm{H} 2 \mathrm{O}} \lesssim 18 \mathrm{~km} \mathrm{~s}^{-1}$, that likely correspond to matter that is being further accelerated before exhibiting $\mathrm{OH}$ masers at $v_{\mathrm{OH}} \sim 26 \mathrm{~km} \mathrm{~s}^{-1}$;

- The first detection of $22 \mathrm{GHz} \mathrm{H}_{2} \mathrm{O}$ maser emission from the cluster supergiant IRAS 05280-6910. It consists of a central emission peak and additional emission components indicating outflows with $v_{\mathrm{H} 2 \mathrm{O}} \sim 6 \mathrm{~km} \mathrm{~s}^{-1}$, that are 
accelerated further before reaching the $\mathrm{OH}$ masing regions that expand with $v_{\mathrm{OH}} \sim 17 \mathrm{~km} \mathrm{~s}^{-1}$;

- The tentative detection of $22 \mathrm{GHz} \mathrm{H}_{2} \mathrm{O}$ maser emission from IRAS 05216-6753, probably a massive star inside an $\mathrm{H}$ II region. No other masers have been detected;

- The tentative detection of $22 \mathrm{GHz} \mathrm{H}_{2} \mathrm{O}$ maser emission from the AGB star IRAS 05329-6708 is puzzling as it suggests an extremely fast outflow of $v \sim 44 \mathrm{~km} \mathrm{~s}^{-1}$;

- Masers in the LMC are generally blue-asymmetric and/or single-peaked. We propose that this may be due to the amplification of stellar and/or free-free radiation, rather than dust emission. This may be more pronounced in low metallicity envelopes due to the low dust content; - There is weak evidence for the expansion velocities in LMC objects to be lower than of similar Galactic objects. The data is also consistent with no difference in expansion velocities, due to the limited sample of LMC objects with reliable estimates of the expansion velocity. The acceleration through the CSE also seems to be slower in LMC objects, with the outflow velocity increasing by a factor of two between the $\mathrm{H}_{2} \mathrm{O}$ and $\mathrm{OH}$ masing zones;

- $\mathrm{SiO}, \mathrm{H}_{2} \mathrm{O}$ and $\mathrm{OH}$ maser emission from circumstellar envelopes in the LMC is found to be equally strong as from similar envelopes in the Milky Way. A larger IR amplitude of variability leads to an increase in the flux density ratio of the $\operatorname{SiO}_{v=1}(J=2 \rightarrow 1)$ peak and the $2.2 \mu \mathrm{m}$ continuum, but the flux density ratio of the $\mathrm{SiO}_{v=1}(J=2 \rightarrow 1)$ peak and the $10 \mu \mathrm{m}$ spectral region is virtually a constant. This suggests a close connection between the shocked $\mathrm{SiO}$ masing region and the dusty outflow, which seems to be similar in the Milky Way and in the LMC.

Present-day facilities for observing $\mathrm{SiO}, \mathrm{H}_{2} \mathrm{O}$ and $\mathrm{OH}$ masers offer an angular resolution and sensitivity capable of detecting only the very brightest masers in the Magellanic Clouds. In the future, ALMA may provide considerably larger samples of Magellanic $\mathrm{SiO}$ and $\mathrm{H}_{2} \mathrm{O}$ masers, but for $1612 \mathrm{MHz} \mathrm{OH}$ masers no major improvement is envisaged. Detection of $\mathrm{H}_{2} \mathrm{O}$ masers in $\mathrm{OH} / \mathrm{IR}$ stars in the Magellanic Clouds would, in principle, allow to derive the total (gas+dust) mass-loss rates. Comparison of $\mathrm{H}_{2} \mathrm{O}$ and $\mathrm{OH}$ deduced expansion velocities yields the acceleration of the radiation-driven wind, if the location of the $\mathrm{H}_{2} \mathrm{O}$ and $\mathrm{OH}$ masers is known with sufficient accuracy. As the distances and hence the luminosities for these stars are known, the gas-to-dust ratios and total mass-loss rates are derived simultaneously (Netzer \& Elitzur 1993).

Acknowledgements. We would like to thank the staff at the SEST, Parkes and Mopra observatories for their kind and helpful support, and in particular Drs. Peter te Lintel Hekkert, Marcus Price and Ian Stewart for help with the $22 \mathrm{GHz}$ observations at Parkes in August 1997. We also thank Dr. Roland Gredel for help with the NTT observations at La Silla in January 1996. We acknowledge the granting of Director's Discretionary Time for obtaining the NTT data. We made use of the SIMBAD database, operated at CDS, Strasbourg, France. We thank the referee Dr. Anders Winnberg for critical comments that helped improve the presentation.

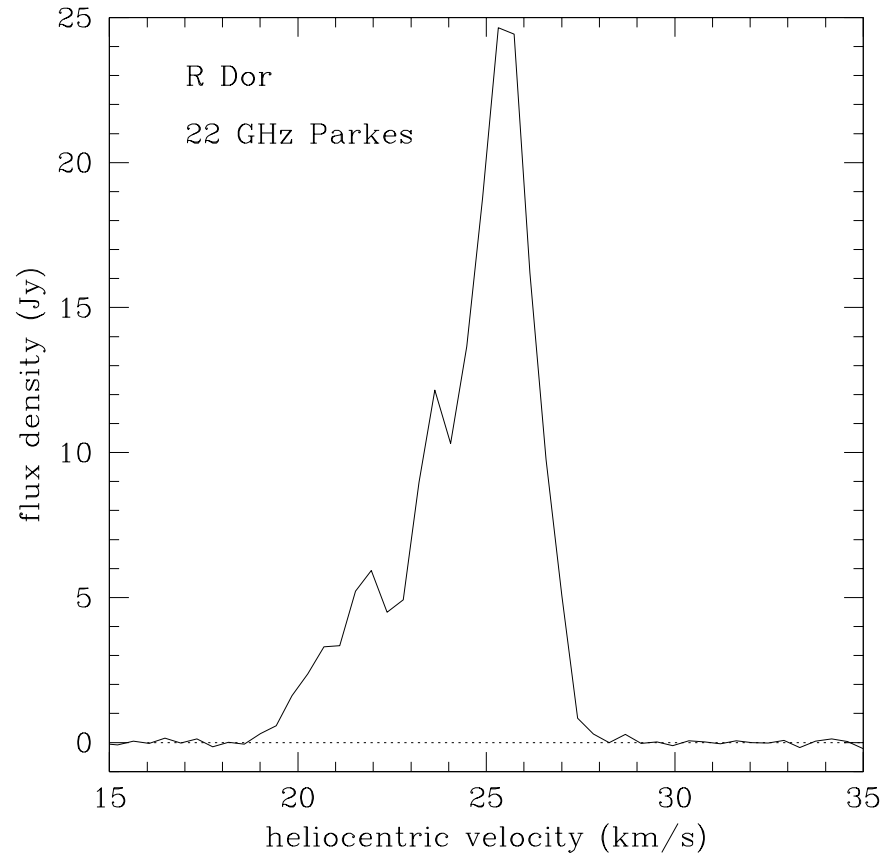

Fig. A.1. R Doradus: Circumstellar 22 GHz (Parkes 2000) $\mathrm{H}_{2} \mathrm{O}$ maser emission. The velocities are heliocentric

Jacco thanks Joana Oliveira for help in reading funny formats of data, improving this manuscript, and much more.

\section{Appendix $\mathrm{A}: \mathrm{H}_{2} \mathrm{O}$ maser emission from the Galactic AGB star R Doradus}

$\mathrm{R}$ Doradus (IRAS 04361-6210: $F_{12}=5157, F_{25}=$ $1594 \mathrm{Jy}$ ) is a famous 6th magnitude ( $V$-band) SRb variable AGB star with a period of 338 days and a spectral type M8 IIIe. It is at a distance of only $61 \mathrm{pc}$, making it the biggest star on the night sky (Bedding et al. 1997 and references therein).

The $22 \mathrm{GHz}$ spectrum with Parkes is presented in Fig. A.1, with an on-source integration time of 228 seconds resulting in an rms noise of $74 \mathrm{mJy}$. The (heliocentric) stellar restframe velocity is at $v_{\star}=+25 \mathrm{~km} \mathrm{~s}^{-1}$. The blueasymmetric $\mathrm{CO}(1 \rightarrow 0)$ line profile indicates an expansion velocity of $v_{\exp }=6.2 \mathrm{~km} \mathrm{~s}^{-1}$ (Lindqvist et al. 1992b) and a mass-loss rate of $\dot{M} \sim 10^{-7} M_{\odot} \mathrm{yr}^{-1}$ (Loup et al. 1993). The $\mathrm{H}_{2} \mathrm{O}$ maser emission appears red-asymmetric, but as the peak very nearly coincides with the stellar velocity there is actually more emission at the blue-shifted rather than at the red-shifted side. Possibly the red-shifted part of the emission is being occulted by the star. The blue-shifted emission extends to (blue-shifted) velocities of almost $\sim 6 \mathrm{~km} \mathrm{~s}^{-1}$ with respect to the stellar restframe. The $\mathrm{H}_{2} \mathrm{O}$ masers thus seem to trace material that includes matter that has already nearly reached the final outflow velocity.

$\mathrm{R}$ Dor is a good example of an AGB star with moderate mass loss. If placed at the distance of the LMC, it would have been barely detectable by ISO in the mid-IR 
(several mJy), and three orders of magnitude too faint to be detectable at $22 \mathrm{GHz}$ (few $\times 10^{-2} \mathrm{mJy}$ ).

\section{Appendix B: Echelle 0.6 to $0.9 \mu \mathrm{m}$ spectra of IRAS 04553-6825}

The $3.5 \mathrm{~m}$ New Technology Telescope (NTT) at the European Southern Observatory (ESO) at La Silla, Chile, was used on October 7, 1995, and January 7, 1996, with the ESO Multi-Mode Instrument (EMMI) to obtain echelle spectra of IRAS 04553-6825. Grating \#14 was used with grism \#6 as cross disperser in October, and grism \#4 in January, yielding a spectral coverage from 6100 to $8300 \AA$ and from 6000 to $9000 \AA$, respectively. The slit width and length were $1^{\prime \prime}$ and $10^{\prime \prime}$ in October, and $2^{\prime \prime}$ and $4^{\prime \prime}$ in January. The total integration time was $1.5 \mathrm{hr}$ in October, and $1 \mathrm{hr}$ in January. The data were reduced in the normal way using the Munich Interactive Data Analysis Software (MIDAS) package. The wavelength calibration was done by taking a ThAr lamp spectrum in conditions identical to the spectrum of IRAS 04553-6825. The measured spectral resolving power is $\sim 7.510^{4}$ for the spectrum taken in October, and $\sim 410^{4}$ for the January spectrum. The average seeing was $\sim 1^{\prime \prime}$ on both nights.

Our echelle spectra resemble the lower resolution spectrum published by Elias et al. (1986), confirming the spectral type of M7.5. Photometry obtained using the $0.9 \mathrm{~m}$ Dutch telescope at ESO, La Silla, yield an approximate average $I$-band magnitude $m_{I} \sim 13$ and $(V-I) \sim$ 6 mag. Van Loon et al. (1998b) concluded on the ba-

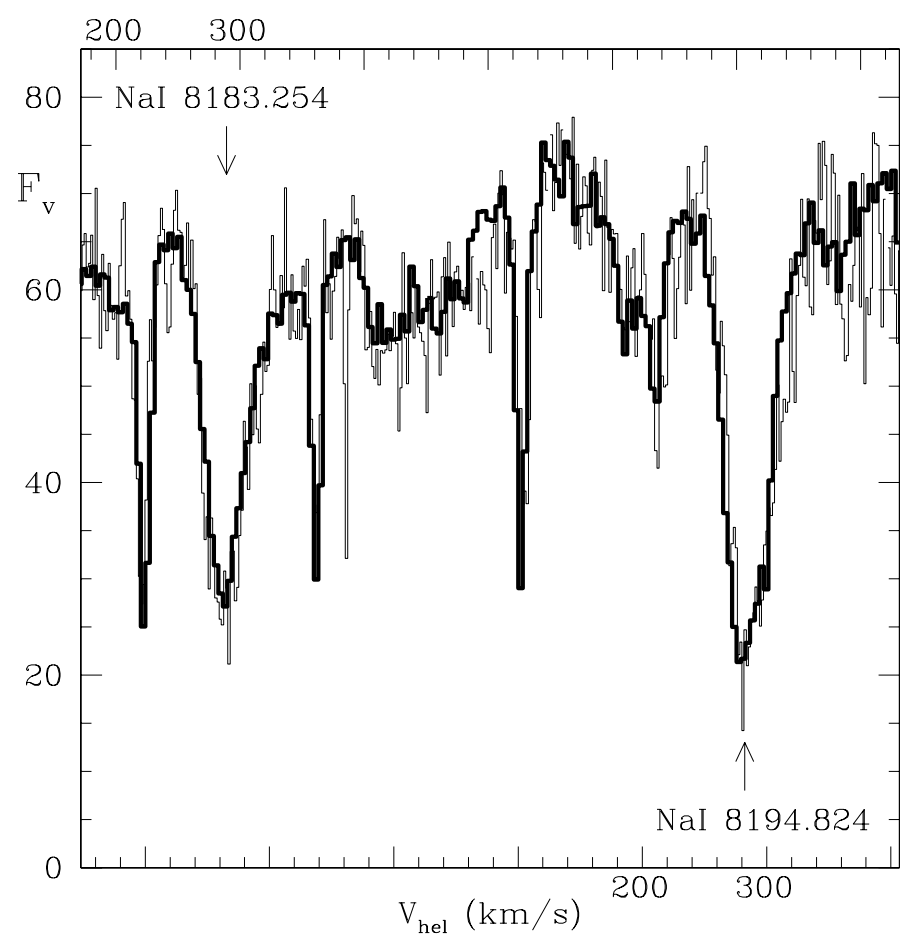

Fig. B.1. NTT echelle spectra of IRAS 04553-6825 (October 1995, plus January 1996 in boldface) around Na I $\lambda \lambda 8183,8195$, with corresponding heliocentric velocity axes. Flux units are arbitrary. The narrow absorption lines are of telluric origin sis of the equivalent widths of the $\mathrm{Ca}$ II triplet lines that IRAS 04553-6825 has a typical LMC metallicity $(Z=0.008)$.

The echelle spectrum of IRAS $04553-6825$ around the $\mathrm{Na}$ I $\lambda \lambda 8183,8195$ lines is presented in Fig. B.1, on an arbitrary flux scale. Note the perfect match between the spectra taken with a time interval of 3 months or 0.1 in pulsation phase, both between minimum and average light in the $K$-band. The absorption is maximum at a heliocentric velocity between 280 and $290 \mathrm{~km} \mathrm{~s}^{-1}$, whereas the K I $\lambda 7699$ and Ca II $\lambda \lambda \lambda 8498,8542,8662$ absorption is maximum at $295 \mathrm{~km} \mathrm{~s}^{-1}$. This is due to scattering of the photospheric spectrum by an extended, expanding dust shell (see Romanik \& Leung 1981, and references therein).

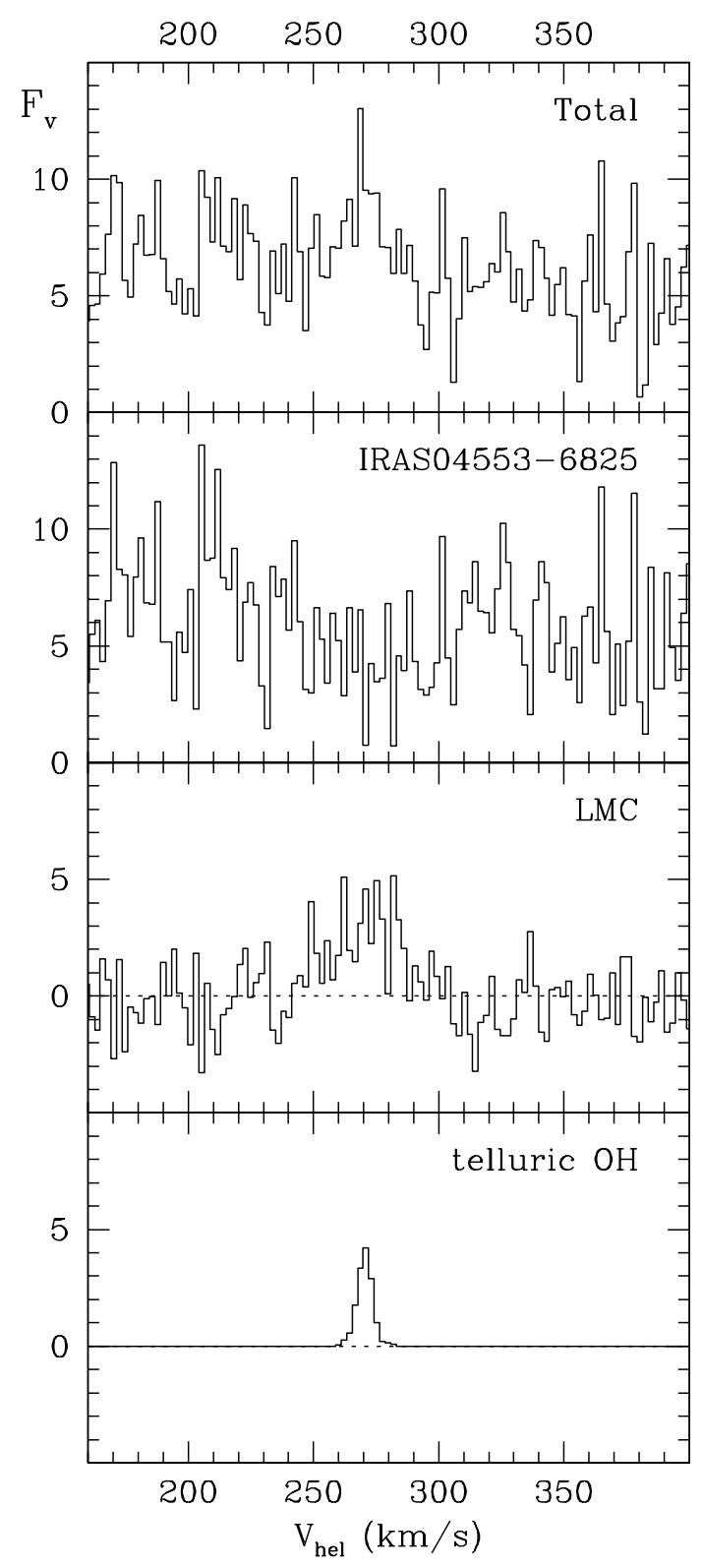

Fig. B.2. NTT echelle spectrum of IRAS 04553-6825 around $\mathrm{H} \alpha$, deconvolved into the contributions of the photosphere of IRAS 04553-6825, the ISM of the LMC, and the telluric OH. Flux units are arbitrary. The heliocentric velocities are for $\mathrm{H} \alpha$ 
The equivalent widths are measured as $W_{8183}=0.72 \pm$ $0.04 \AA$ and $W_{8195}=0.88 \pm 0.04 \AA$ in October 1995 , and $W_{8183}=0.66 \pm 0.03 \AA$ and $W_{8195}=0.82 \pm 0.02 \AA$ in January 1996. Their sum is on average $W_{8183+8195}=$ $1.51 \pm 0.04 \AA$. The equivalent widths of the $\mathrm{Na}$ I lines reach their minima in giants, and are larger in both dwarfs and supergiants. Comparison with Schiavon et al. (1997), and excluding a dwarf status, confirms that IRAS 04553-6825 has a supergiant-type optical spectrum.

Elias et al. (1986) claim the association with $\mathrm{H} \alpha$ and forbidden line emission from $\mathrm{N}^{+}$and $\mathrm{S}^{+}$ions. However, the red-shift of IRAS $04553-6825$ is such that the expected observed wavelengths of both the $\mathrm{H} \alpha, \mathrm{N}$ II $\lambda 6548$, and S II $\lambda \lambda 6717,6731$ emission lines coincide within $\sim 20 \mathrm{~km} \mathrm{~s}^{-1}$ with telluric $\mathrm{OH}$ emission lines. A stretch of $\sim 10 \AA$ of photospheric spectrum around $6590 \AA$ that is relatively free of molecular absorption is easily mistaken for the N II $\lambda 6583$ line in a lower resolution spectrum like that of Elias et al.

Other telluric $\mathrm{OH}$ lines in the spectrum were used to derive an empirical relation between the intensity and quantum numbers associated with the vibrational and rotational levels of the transition, making use of Osterbrock \& Martel (1992) for identifications. A template line shape of the telluric $\mathrm{OH}$ line was created by averaging 20 bright isolated lines. The telluric line was then subtracted from the spectrum of the star (Fig. B.2). What remains is spatially extended $\mathrm{H} \alpha$ emission covering $\sim 50 \mathrm{kms}^{-1}$, and weak photospheric $\mathrm{H} \alpha$ absorption. A $10 \mathrm{~min}$ exposure taken through an $\mathrm{H} \alpha$ filter with the NTT of the region around IRAS 04553-6825 (http://www.eso.org/outreach/press-rel/pr-1996/ phot-15-96.gif) shows extended nebular complexes a few arcmin SW and faint filamentary emission due $\mathrm{E}$ of the star, but no $\mathrm{H} \alpha$ emission that can be attributed to the emission detected in the spectrum at $\sim 1$ pc from the star.

\section{Appendix C: Galactic Centre masers}

Wood et al. (1998), Blommaert et al. (1998) and Ortiz et al. (2000) have searched for near- and mid-IR counterparts of $\mathrm{OH}$ sources in the direction of the Galactic Centre, mainly from Lindqvist et al. (1992a). Their studies are suitable for comparison of the properties of maser emission, expansion velocities, pulsation periods and amplitudes, and CSE optical depths. A distance to the Galactic Centre of $8 \mathrm{kpc}$ is adopted (Reid 1993).

Cross-identifications of the samples of stars in Lindqvist et al. (1992a) and Wood et al. (1998) with the IRAS Point Source Catalogue (PSC) are summarised in Table C.1. Where the $\mathrm{OH}$ source and/or LPV lies inside of the error ellipse as given in the PSC, the identification was tagged positive $(+)$. If the distances along both axes of the error ellipse are less than $3 \sigma$, the identification was tagged questionable (?). This resulted in 11 likely IRAS counterparts. All of these that have known near-IR counterparts have IR colours that suggest oxygen-rich CSEs (from $(K-[12])$ versus $(H-K)$, see van Loon et al. 1998a).
The identification of 65.119 with IRAS $17424-2859$ is uncertain because the $12 \mu \mathrm{m}$ emission is much too bright to arise from a CSE around an evolved star given its near-IR colours, and this object is further ignored. Ortiz et al. (2000) searched for 7 and $15 \mu \mathrm{m}$ counterparts of $\mathrm{OH} / \mathrm{IR}$ stars in the Galactic Centre using the ISOGAL (Omont et al. 1999) database. All of their identifications of Lindqvist sources with IRAS sources are also recovered by us, but there are at least as many more identifications that they did not find. The ISOGAL survey had to avoid strong IRAS point sources and is therefore biased, whereas the IRAS survey and our approach of identifying $\mathrm{OH} / \mathrm{IR}$ stars are expected to be more homogeneous.

The IRAS $12 \mu \mathrm{m}$ flux densities of the positively identified sources are all in the range 2.8 to $12 \mathrm{Jy}$. This corresponds to flux densities from 0.07 to $0.3 \mathrm{Jy}$ at the distance of the LMC. OH/IR stars with IRAS $12 \mu \mathrm{m}$ flux densities corresponding to $\sim 1 \mathrm{Jy}$ at the LMC are not encountered in the Galactic Centre, but do exist in the MCs (e.g. Table 2). This is especially surprising as it has been suggested that among the Galactic Centre $\mathrm{OH} / \mathrm{IR}$ stars are massive, metal-rich mass-losing LPVs that are expected to have a large IR excess emission from their dusty CSEs. At the other extreme, if most of the Galactic Centre CSEs have $12 \mu \mathrm{m}$ flux densities lower than the positively identified IRAS sources, they would have been largely undetected by IRAS in the MCs.

Wood et al. (1998) find LPVs with $K$-band magnitudes from 5 to 13 after correction for interstellar extinction, with the faintest IRAS associated LPV to have $K_{0}=$ 10.33 mag. At the distance of the LMC this would yield $K$-band magnitudes from 9 to 17 , so most of these would also have been found by the IRAC2 searches for nearIR counterparts of IRAS sources in the MCs by Zijlstra et al. (1996), van Loon et al. (1997), and Groenewegen \& Blommaert (1998). The $(H-K)_{0}$ colours of the Galactic Centre LPVs are $\sim 2$ mag on average and $\sim 4$ mag maximum, similar to the $(H-K)$ colours of the obscured AGB stars in the MCs.

The mean values of $\log (P)$ and $M_{\mathrm{bol}}$ according to the $(K-L)_{0}$ colour (corrected for interstellar extinction) for the LPVs in the Galactic Centre follow a sequence in the period-luminosity (P-L) diagram (Fig. C.1). Also plotted are the $\mathrm{OH}$ maser sources in the LMC that have known pulsation periods and bolometric magnitudes, and the P-L relations that have been derived from samples of oxygenrich LPVs (dotted) and carbon-rich LPVs (dashed) with periods $P<420 \mathrm{~d}$ (Feast et al. 1989). The LMC OH maser stars with known pulsation period have typically $(K-L) \sim 2$ mag. Yet they appear to be on the extension of the $(K-L)$ colour sequence as traced by the Galactic Centre LPVs, despite that this sequence already reaches $(K-L)>4$ mag before reaching the long periods and high luminosities of the LMC stars. This illustrates that LMC stars with a similar stellar structure as Galactic Centre stars have less dusty CSEs due to their lower metallicity (see also van Loon 2000). 
Table C.1. Identification of Galactic Centre AGB stars in the IRAS Point Source Catalogue. Identifiers (L)WHM are from Lindqvist et al. (1992a) and Wood et al. (1998), where the prefix S refers to single-peaked OH masers. The PSC entry of a spatially coincident IRAS source is given, together with the differences in Right Ascension $(\alpha)$ and Declination $(\delta)$, and the total distance $\Delta$. The error ellipse of the IRAS detection is given by major and minor axes $\sigma_{\mathrm{a}}$ and $\sigma_{\mathrm{b}}$ and angle $\theta$ indicating the orientation of the major axis, from North over East (i.e. in these cases the major axis is roughly parallel to $\alpha$ ). Identification (id) is decided positive $(+)$ in case of agreement within the error ellipse, and questionable (?) if not more than $3 \sigma$ distance. Where available, we quote from Lindqvist et al. (1992a) and Wood et al. (1998) the interstellar extinction corrected $(H-K)$ and $K$ photometry, IRAS-PSC 12,25 , and $60 \mu$ m flux densities, bolometric magnitude $M_{\text {bol }}$, pulsation period $P$, and OH derived expansion velocity $v_{\infty}$ of the CSE. Underlined names are sources with ISOGAL counterparts found by Ortiz et al. (2000)

\begin{tabular}{|c|c|c|c|c|c|c|c|c|c|c|c|c|c|c|c|c|}
\hline$\overline{\text { (L)WHM }}$ & IRAS-PSC & 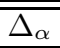 & $\overline{\overline{\Delta_{\delta}}}$ & $\overline{\overline{\Delta \Delta}}$ & $\overline{\overline{\sigma_{\mathrm{a}}}}$ & $\overline{\sigma_{\mathrm{b}}}$ & $\overline{\bar{\theta}}$ & $\overline{\text { id }}$ & $\overline{(\overline{(H-K)}}$ & $\bar{~} \bar{K}$ & $\overline{S_{12}}$ & $\overline{S_{25}}$ & 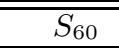 & 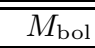 & $\overline{\bar{P}}$ & $v_{\infty}$ \\
\hline 5. & $17421-2931$ & 3 & 2 & 3 & 26 & 5 & 93 & + & & & 3.3 & 5.2 & $<30$ & & & 17.6 \\
\hline 13. & $17423-2924$ & 9 & 8 & 12 & 34 & 6 & 93 & $?$ & & & 4.7: & 16 & $<362$ & & & 14.2 \\
\hline 13.015 & $17423-2924$ & 33 & 16 & 36 & 34 & 6 & 93 & $?$ & 1.29 & 9.43 & 4.7: & 16 & $<362$ & -2.33 & 213 & \\
\hline$\underline{21} .031$ & $17413-2909$ & 1 & 1 & 2 & 31 & 7 & 93 & + & 3.02 & 9.27 & 6.5 & $<52$ & $<606$ & -5.26 & 698 & 18.8 \\
\hline$\underline{33}$. & $\overline{17413-2903}$ & 29 & 2 & 29 & 28 & 6 & 93 & + & & & 9.0 & $<16$ & $<42$ & & & 15 . \\
\hline$\underline{34} .004$ & $\overline{17419-2907}$ & 37 & 27 & 46 & 26 & 13 & 93 & $?$ & 0.88 & 7.14 & 4.6 & 117 & 467: & -4.69 & 453 & 14.2 \\
\hline$\underline{34.063}$ & $\underline{17419-2907}$ & 55 & 9 & 56 & 26 & 13 & 93 & $?$ & 2.52 & 9.60 & 4.6 & 117 & 467: & -3.70 & 915 & \\
\hline 39.001 & $\overline{17433-2918}$ & 49 & 2 & 49 & 31 & 6 & 93 & $?$ & 0.82 & 7.12 & $<7.3$ & 6.3 & $<77$ & -4.25 & 355 & \\
\hline 39.002 & $17433-2918$ & 50 & 5 & 50 & 31 & 6 & 93 & $?$ & 2.23 & 7.53 & $<7.3$ & 6.3 & $<77$ & -4.69 & 559 & 13.3 \\
\hline 60.001 & $17421-2857$ & 4 & 13 & 14 & 19 & 5 & 93 & $?$ & 1.02 & 6.13 & 16 & 298 & $<4318$ & -5.25 & 576 & \\
\hline$\underline{64} .028$ & $\underline{17412-2849}$ & 7 & 2 & 7 & 22 & 6 & 93 & + & 2.25 & 8.42 & 4.1 & 5.8 & $<56$ & -5.70 & 692 & 17.0 \\
\hline 65.119 & $\overline{17424-2859}$ & 4 & 6 & 7 & 22 & 7 & 93 & + & 1.86 & 8.07 & 1360 & 5147 & 18560 & -5.01 & 799 & 19.9 \\
\hline 71.002 & $17417-2851$ & 29 & 7 & 30 & 31 & 9 & 93 & + & 2.82 & 9.38 & 11: & 54 & 671: & -4.76 & 636 & 24.4 \\
\hline$\underline{79} .001$ & $\underline{17411-2843}$ & 30 & 3 & 30 & 32 & 5 & 102 & + & 1.07 & 6.48 & 3.4 & 4.2 & $<53$ & -5.02 & 477 & 9.7 \\
\hline$\overline{82.007}$ & $\overline{17428-2854}$ & 16 & 2 & 16 & 23 & 7 & 93 & + & 1.83 & 7.99 & 12 & 151: & $<57$ & -4.89 & 701 & 23.9 \\
\hline$\underline{101.014}$ & $\underline{17419-2837}$ & 61 & 1 & 61 & 74 & 7 & 93 & + & 3.41 & 10.33 & $<10$ & 4.3 & $<99$ & -4.98 & 825 & 15.8 \\
\hline 134 . & $\overline{17436-2807}$ & 1 & 8 & 8 & 47 & 5 & 93 & $?$ & & & 4.7 & $<30$ & $<451$ & & & 16.3 \\
\hline$\underline{\mathrm{S} 02}$. & $17428-2918$ & 39 & 1 & 39 & 46 & 10 & 92 & + & & & $2.8:$ & 6.3 & $<212$ & & & \\
\hline$\underline{\mathrm{S} 07}$. & $17429-2903$ & 55 & 17 & 58 & 30 & 7 & 102 & $?$ & & & $<7.6$ & 23 & 548: & & & \\
\hline$\underline{\mathrm{S} 15}$. & $17441-2822$ & 7 & 3 & 7 & 23 & 6 & 93 & + & & & $<7.7$ & 69 & 12952 & & & \\
\hline
\end{tabular}

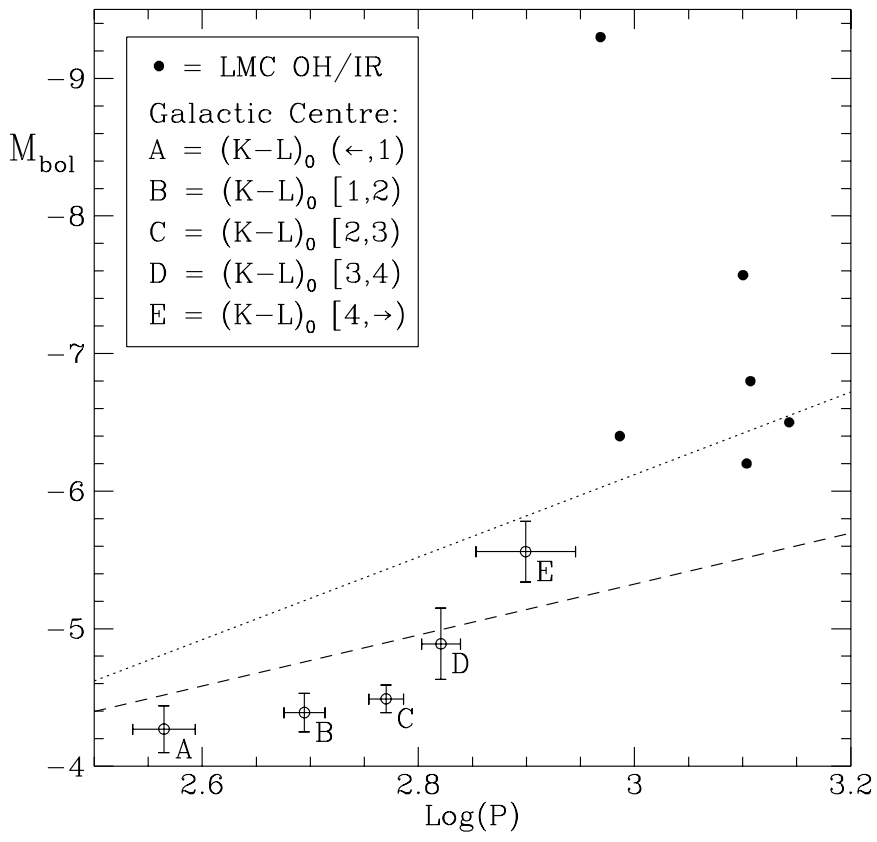

Fig. C.1. Period-luminosity diagram for $\mathrm{OH} / \mathrm{IR}$ stars in the LMC and Galactic Centre - the latter grouped according to near-IR colour. The lines are relations derived from oxygen (dotted) and carbon-rich (dashed) LPVs with $P<420 \mathrm{~d}$

\section{References}

Alcolea, J., Bujarrabal, V., \& Gómez-González, J. 1990, A\&A, 231,431
Alcolea, J., Pardo, J. R., Bujarrabal, V., et al. 1999, A\&AS, 139,461

Bedding, T. R., Zijlstra, A. A., van der Lühe, O., et al. 1997, MNRAS, 286, 957

Bica, E. L. D., Schmitt, H. R., Dutra, C. M., \& Oliveira, H. L. 1999, AJ, 117, 238

Blommaert, J. A. D. L., van der Veen, W. E. C. J., van Langevelde, H. J., Habing, H. J., \& Sjouwerman, L. O. 1998, A\&A, 329, 991

Bohannan, B., \& Epps, H. W. 1974, A\&AS, 18, 47

Bujarrabal, V., Alcolea, J., Sánchez-Contreras, C., \& Colomer, F. 1996, A\&A, 314, 883

Castilho, B. V., Gregorio-Hetem, J., Spite, F., Spite, M., \& Barbuy, B. 1998, A\&AS, 127, 139

Cernicharo, J., Alcolea, J., Baudry, A., \& González-Alfonso, E. 1997, A\&A, 319, 607

Cioni, M.-R., Loup, C., \& Habing, H. J. 2000, A\&AS, 144, 235

Colomer, F., Reid, M. J., Menten, K. M., \& Bujarrabal, V. 2000, A\&A, 355, 979

Diamond, P. J., Norris, R. P., \& Booth, R. S. 1984, MNRAS, 207,611

Elias, J. H., Frogel, J. A., \& Schwering, P. B. W. 1986, ApJ, 302,675

Feast, M. W., Glass, I. S., Whitelock, P. A., \& Catchpole, R. M. 1989, MNRAS, 241, 375

Fehrenbach, C., \& Duflot, M. 1982, A\&AS, 48, 409

Goldreich, P., \& Scoville, N. Z. 1976, ApJ, 205, 144

González-Alfonso, E., Cernicharo, J., Alcolea, J., \& Orlandi, M. A. 1998, A\&A, 334, 1016

Groenewegen, M. A. T., \& Blommaert, J. A. D. L. 1998, A\&A, 332,25 
Groenewegen, M. A. T., Smith, C. H., Wood, P. R., Omont, A., \& Fujiyoshi, T. 1995, ApJ, 449, L119

Groenewegen, M. A. T., Whitelock, P. A., Smith, C. H., \& Kerschbaum, F. 1998, MNRAS, 293, 18

Haikala, L. K. 1990, A\&AS, 85, 875

Haikala, L. K., Nyman, L. Å., \& Forsström, V. 1994, A\&AS, 103, 107

Harvey, P. M., Bechis, K. P., Wilson, W. J., \& Ball, J. A. 1974, ApJS, 27, 331

Henize, K. G. 1956, ApJS, 2, 315

Herpin, F., Baudry, A., Alcolea, J., \& Cernicharo, J. 1998, A\&A, 334, 1037

Huggins, P. J., \& Glassgold, A. E. 1982, AJ, 87, 1828

Izumiura, H., Deguchi, S., Hashimoto, O., et al. 1994, ApJ, 437,419

Kim, S., Dopita, M. A., Staveley-Smith, L., \& Bessell, M. S. 1999, AJ, 118, 2797

Kwok, S., Volk, K., \& Bidelman, W. P. 1997, ApJS, 112, 557

Laval, A., Rosado, M., Boulesteix, J., et al. 1992, A\&A, 253, 213

Laval, A., Gry, C., Rosado, M., Marcelin, M., \& Greve, A. 1994, A\&A, 288, 572

Le Bertre, T. 1993, A\&AS, 97, 729

Le Bertre, T., \& Nyman, L.-A. 1990, 233, 477

Lewis, B. M. 1989, ApJ, 338, 234

Lewis, B. M. 1991, AJ, 101, 254

Lewis, B. M. 1998, ApJ, 508, 831

Lindqvist, M., Winnberg, A., \& Forster, J. R. 1990, A\&A, 229, 165

Lindqvist, M., Ukita, N., Winnberg, A., \& Johansson, L. E. B. 1991, A\&A, 250, 431

Lindqvist, M., Habing, H. J., \& Winnberg, A. 1992a, A\&A, 259, 118

Lindqvist, M., Olofsson, H., Winnberg, A., \& Nyman, L.1992b, A\&A, 263, 183

Loup, C., Forveille, T., Omont, A., \& Paul, J. F. 1993, A\&AS, 99, 291

Loup, C., Zijlstra, A. A., Waters, L. B. F. M., \& Groenewegen, M. A. T. 1997, A\&AS, 125, 419

Luks, Th., \& Rohlfs, K. 1992, A\&A, 263, 41

Meaburn, J. 1980, MNRAS, 192, 365

Meyssonnier, N., \& Azzopardi, M. 1993, A\&AS, 102, 451

Morris, M., \& Jura, M. 1983, ApJ, 267, 179

Netzer, N., \& Elitzur, M. 1993, ApJ, 410, 701

Norris, R. P., Booth, R. S., Diamond, P. J., et al. 1984, MNRAS, 208, 435

Nyman, L.-A., \& Olofsson, H. 1985, A\&A, 147, 309

Nyman, L.-A., \& Olofsson, H. 1986, A\&A, 158, 67

Nyman, L.-Å, Hall, P. J., \& Le Bertre, T. 1993, A\&A, 280, 551

Nyman, L.-Å, Hall, P. J., \& Olofsson, H. 1998, A\&AS, 127, 185

Omont, A., The ISOGAL Collaboration 1999, in The Universe as Seen by ISO, ed. P. Cox, \& M. F. Kessler., ESA-SP, 427, 211

Ortiz, R., Blommaert, J. A. D. L., Copet, E., et al. 2000, submitted to A\&A

Osterbrock, D. E., \& Martel, A. 1992, PASP, 104, 76

Parker, J. W., Garmany, C. D., Massey, P., \& Walborn, N. R. 1992, AJ, 103, 1205

Persi, P., Palagi, F., \& Felli, M. 1994, A\&A, 291, 577

Prévot, L., Rousseau, J., \& Martin, N. 1989, A\&A, 225, 303
Rebeirot, E., Azzopardi, M., \& Westerlund, B. E. 1993, A\&AS, 97, 603

Reid, M. J. 1993, ARA\&A, 31, 345

Reid, I. N., Tinney, C. G., \& Mould, J. R. 1990, ApJ, 348, 98

Richards, A. M. S., Yates, J. A., \& Cohen, R. J. 1996, MNRAS, 282,665

Romanik, C. J., \& Leung, C. M. 1981, ApJ, 246, 935

Rosado, M., Laval, A., Le Coarer, E., et al. 1996, A\&A, 308, 588

Schiavon, R. P., Barbuy, B., Rossi, S. C. F., \& Milone, A. 1997, ApJ, 479, 902

Sivagnanam, P., \& David, P. 1999, MNRAS, 304, 622

Sivagnanam, P., Le Squeren, A. M., Foy, F., \& Tran Minh, F. 1989, A\&A, 211, 341

Sivagnanam, P., Le Squeren, A. M., Biraud, F., \& Diamond, P. J. 1990, A\&A, 229, 171

Sjouwerman, L. O., van Langevelde, H. J., Winnberg, A., \& Habing, H. J. 1998, A\&AS, 128, 35

Smith, V. V., Plez, B., \& Lambert, D. L. 1995, ApJ, 441, 735

Stanimirović, S., Staveley-Smith, L., Dickey, J. M., Sault, R. J., \& Snowden, S. L. 1999, MNRAS, 302, 417

Staveley-Smith, L., Sault, R. J., Hatzidimitriou, D., Kesteven, M., \& McConnell, D. 1997, MNRAS, 289, 225

Takaba, H., Ukita, N., Miyaji, T., \& Miyoshi, M. 1994, PASJ, 46,629

Trams, N. R., van Loon, J. Th., Waters, L. B. F. M., et al. 1999, A\&A, 346, 843

van Loon, J. Th. 2000, A\&A, 354, 125

van Loon, J. Th., \& Zijlstra, A. A. 2000, ApJL, in press

van Loon, J. Th., Zijlstra, A. A., Bujarrabal, V., \& Nyman, L.-Å. 1996, A\&A, 306, L29

van Loon, J. Th., Zijlstra, A. A., Whitelock, P. A., et al. 1997, A\&A, 325, 585

van Loon, J. Th., Zijlstra, A. A., Whitelock, P. A., et al. 1998a, A\&A, 329, 169

van Loon, J. Th., te Lintel Hekkert, P., Bujarrabal, V., Zijlstra, A. A., \& Nyman, L.-A 1998b, A\&A, 337, 141

van Loon, J. Th., Zijlstra, A. A., \& Groenewegen, M. A. T. 1999a, A\&A, 346, 805

van Loon, J. Th., Groenewegen, M. A. T., de Koter, A., et al. 1999b, A\&A, 351, 559

van Loon, J. Th., Zijlstra, A. A., Kaper, L., et al. 2000, A\&A, in press

Westerlund, B. E., Olander, N., \& Hedin, B. 1981, A\&AS, 43, 267

Whitelock, P. A., Feast, M. W., Menzies, J. W., \& Catchpole, R. M. 1989, MNRAS, 238, 769

Whiteoak, J. B., \& Gardner, F. F. 1986, MNRAS, 222, 513

Wood, P. R. 1998, A\&A, 338, 592

Wood, P. R., Bessell, M. S., \& Fox, M. W. 1983, ApJ, 272, 99

Wood, P. R., Bessell, M. S., \& Whiteoak, J. B. 1986, ApJ, 306, L81

Wood, P. R., Whiteoak, J. B., Hughes, S. M. G., et al. 1992, ApJ, 397, 552

Wood, P. R., Habing, H. J., \& McGregor, P. J. 1998, A\&A, 336, 925

Yates, J. A., Field, D., \& Gray, M. D. 1997, MNRAS, 285, 303

Zijlstra, A. A., Loup, C., Waters, L. B. F. M., et al. 1996, MNRAS, 279, 32

Zijlstra, A. A., Chapman, J. M., te Lintel Hekkert, P., et al. 2000, MNRAS, in press 